\title{
An Efficient Two-Layer Non-hydrostatic Approach for Dispersive Water Waves
}

\author{
C. Escalante $^{1}$ • E. D. Fernández-Nieto ${ }^{2} \quad$-T. Morales de Luna ${ }^{3} \quad$ • M. J. Castro ${ }^{1}$
}

\begin{abstract}
In this paper, we propose a two-layer depth-integrated non-hydrostatic system with improved dispersion relations. This improvement is obtained through three free parameters: two of them related to the representation of the pressure at the interface and a third one that controls the relative position of the interface concerning the total height. These parameters are then optimized to improve the dispersive properties of the resulting system. The optimized model shows good linear wave characteristics up to $k H \approx 10$, that can be improved for long waves. The system is solved using an efficient formally second-order well-balanced and positive preserving hybrid finite volume/difference numerical scheme. The scheme consists of a two-step algorithm based on a projection-correction type scheme. First, the hyperbolic part of the system is discretized using a Polynomial Viscosity Matrix path-conservative finite-volume method. Second, the dispersive terms are solved using finite differences. The method has been applied to idealized and challenging physical situations that involve nearshore breaking. Agreement with laboratory data is excellent. This technique results in an accurate and efficient method.
\end{abstract}

Keywords Dispersive waves · Non-hydrostatic · Shallow-water · Finite-volume · Finite-difference $\cdot$ Breaking waves

\section{Introduction}

When modelling and simulating geophysical flows, the non-linear shallow-water equations, from now on SWE, is often a good choice as an approximation of the Navier-Stokes equations. Nevertheless, SWE does not take into account effects associated with dispersive waves. In recent years, a great effort has been made in the derivation of relatively simple mathematical models for shallow water flows that include long non-linear water waves. As computational power increases, Boussinesq type models [1,15,40,52,58,59,67-69] become more accessible.

\footnotetext{
C. Escalante

escalante@uma.es

1 Universidad de Málaga, Málaga, Spain

2 Universidad de Sevilla, Seville, Spain

3 Universidad de Córdoba, Córdoba, Spain
} 
This means that one can use more sophisticated models to accurately describe reality, despite the higher computational cost.

Moreover, to improve the non-linear dispersive properties of the model, information on the vertical structure of the flow should be included. The Boussinesq-type wave equations have prevailed due to their computational efficiency. The main idea is to include non-hydrostatic effects due to the vertical acceleration of the fluid in the depth-averaging process of the equations. For instance, one can assume that both non-linearity and frequency dispersion are weak and of the same order of magnitude. Since the early works of Peregrine [59], several improved and enhanced Boussinesq models have been proposed over the years: Madsen and Sørensen [52], Nwogu [58], Serre Green-Naghdi equations [40], and nonlinear and non-hydrostatic higher order shallow-water type models [16,22,34,71] among many others. One may use different approaches to improve non-linear dispersive properties of the models: considering a Taylor expansion of the velocity potential in powers of the vertical coordinate and in terms of the depth-averaged velocity [52] or the particle velocity components $(u, w)$ at a chosen level [58]; including two scalars representing the vertical profile of the non-hydrostatic pressure [42]; using a better flow resolution in the vertical direction with a multi-layer approach [46,47,70].

Weakly-dispersive weakly-nonlinear Boussinesq systems ([16,52,58,71] among others) may offer good dispersive properties such as phase, group velocity and shoaling for $k H$ values up to 3 , where $k$ denotes the wave number and $H$ the typical depth. In intermediate waters, this range could not be enough, and much more information on the vertical structure must be retained. High order Boussinesq equations can offer better linear and non-linear dispersive properties. The counterpart is that extremely complex systems with high order derivatives arise (fifth order derivatives in [38] for $k H$ up to 6), requiring an equally complex numerical scheme. The complexity increases for a two-horizontal dimension problem.

The development of non-hydrostatic models for coastal water waves has been the topic of many studies over the past 15 years. Non-hydrostatic models are capable of solving many relevant features of coastal water waves, such as dispersion, non-linearity, shoaling, refraction, diffraction, and run-up. For example, in [22], Casulli splits the pressure into a hydrostatic and a non-hydrostatic part. Then, the system is approximated with a fractional step method, wherein the first step the hydrostatic free-surface flow model is approximated and in a second step a Poisson equation is solved.

The decomposition of the pressure into a hydrostatic and non-hydrostatic parts is also considered in many other works. This decomposition has been considered for example by Ma et al. [50] and Gallerano et al. [37], where the system is reformulated in $\sigma$-coordinates. Stansby and Zhou [63] also considered this decomposition, where the main difference is that the $\sigma$-coordinate is not considered for the horizontal non-hydrostatic pressure gradients, to avoid the numerical truncate errors introduced by this approximation.

Zijlema and Stelling, in [24,64,72], propose a multi-layer method based in a layered depth-averaging of the system. A similar procedure has been considered by Ai and Jin (see $[4,5])$. The main differences reside in the horizontal gradient of the non-hydrostatic pressure component, which is assumed to be independent of the depth at each layer. Another multi-layer model has been proposed in [10] where a numerical study of the linear dispersive celerity shows convergence to the results given by the linear Stokes theory. The linear shoaling properties of this model are studied in [11]. In [34], a hierarchy of layer-averaged multi-layer models has been proposed based on the hypothesis made for the vertical velocity and pressure profiles. Moreover, an explicit linear dispersion relation for each one is done. This allows proving analytically that when the number of layer increases, celerity converges to the exact one given by Euler equations in the Stokes linear theory. 
Bai and Cheung [9] proposed a modification of the depth-integrated two-layer model introduced in [8]. They include a free-parameter that relates the pressures at each of the two layers. This parameter allows improving the linear dispersion relation. Cui et al. introduced in [27] a two-layer model based in a depth-averaging of the system written in $\sigma$-coordinates. They introduced two free-parameters that relate the height of the two layers and the nonhydrostatic pressures unknowns of the two-layer system. The choice of these parameters is also related to the accuracy of the linear dispersive properties of the system.

The paper is organized as follows. In Sect. 2 we present a new two-layer depth-averaging system depending on three different free-parameters that are optimized to improve the dispersive relation of the system. The main hypothesis for the derivation is the assumption of a constant vertical profile of the horizontal velocity within each layer. This corresponds to the assumption of a shallow water regime as it is usual when deriving non-hydrostatic multilayer systems.

The proposed two-layer system can be seen as a modification of one of the models presented in [34] with a correction in the non-hydrostatic pressure profile. This will leads to a system that improves the main dispersive properties of other two-layer non-hydrostatic pressure systems proposed in the literature (see for instance [27]), and can even improve the dispersive properties of some multi-layer systems with 5 layers (see for instance [11]). We recall that the resulting system is a model which is less expensive from the computational point of view while keeping good dispersive relations. Thus, the final model can be applied to intermediate waters for a wide range of waves for a $k H$ up to 10 . This is discussed through a linear analysis in Sect. 3, as well as numerical tests. It is worth mentioning that this approach leads to a system that does not present those aforementioned high order derivatives.

In Sect. 4 a numerical scheme is introduced based on a two-step projection-correction type scheme. In the first step we solve the hyperbolic hydrostatic system and in the second one, we include the non-hydrostatic effects. The proposed numerical scheme present a novel treatment of wet-dry fronts, that is very robust, as it will be shown in Sect. 6. No special treatment is required for the non-hydrostatic pressure since in the presence of wet-dry fonts it vanishes automatically thanks to the rewriting of the incompressibility equations. This is an improvement on non-hydrostatic pressure numerical schemes, where usually non-hydrostatic pressure is truncated to zero up to a threshold value.

The reader should keep in mind that detailed small-scale breaking driven physics are not described by the model. This means that one has to include some breaking mechanism in the depth-integrated equations. In Sect. 5, a new robust and straightforward breaking mechanism is presented. The breaking mechanism consists in the extension to the two-layer system of the viscous mechanism used in [33]. The breaking criteria used here to switch on/off the breaking mechanism is a simplification of the criteria presented in [61] and is one of the simplest that can be found in the literature. Nevertheless some other and more sophisticated breaking criteria could be considered and implemented similarly.

Finally, in Sect. 6, some numerical tests including comparisons with analytical solutions from the Stokes linear theory and laboratory data are shown.

\section{The Non-hydrostatic Two-Layer System}

In this section, a two layer-averaged system derived from Euler equations is presented. This model is obtained by setting a non-material interface that separates two layers with different velocities and pressures. In the deduction of the equations is assumed that the horizontal 
velocity has a piecewise constant vertical profile, by while the vertical velocity and the nonhydrostatic counterpart pressure are piecewise linear, being the three unknowns discontinuous at the interface.

The 2D $(x, z)$ Euler system reads

$$
\left\{\begin{array}{l}
\partial_{x} u+\partial_{z} w=0 \\
\partial_{t} u+\partial_{x}\left(u^{2}+p_{T}\right)+\partial_{z}(u w)=0 \\
\partial_{t} w+\partial_{x}(u w)+\partial_{z}\left(w^{2}+p_{T}\right)=-g
\end{array}\right.
$$

where $x$ and $z$ denote the horizontal $O x$ and the vertical $O z$ axis respectively. We consider this system for

$$
t>t_{0}, x \in \mathbb{R},-H(x, t) \leq z \leq \eta(x, t),
$$

where $\eta$ is the unknown water elevation, $H$ is the bathymetry that can vary in space and time, $u$ and $w$ are the horizontal and vertical velocities. The water height is $h=\eta+H$. The model is completed with boundary conditions at the free surface

$$
\left\{\begin{array}{l}
\partial_{t} \eta(x, t)+u(x, \eta(x, t), t) \partial_{x} \eta(x, t)-w(x, \eta(x, t), t)=0, \\
p_{T}((x, \eta(x, t), t))=p^{a t m}
\end{array}\right.
$$

where $p^{a t m}$ is the atmospheric pressure. At the bottom, no-penetration boundary condition is imposed

$$
u(x,-H(x, t), t) \partial_{x} H+w(x,-H(x, t), t)+\partial_{t} H=0 .
$$

The total pressure $p_{T}$ is supposed to be decomposed into a sum of a hydrostatic and nonhydrostatic part:

$$
p_{T}=p^{a t m}+g(\eta-z)+p
$$

where $p(x, z, t)$ is the non-hydrostatic pressure. Hereinafter, the atmospheric pressure will be supposed to be zero and the non-hydrostatic pressure is assumed to vanish at the free surface

$$
p(x, \eta(x, t), t)=0 .
$$

Given a positive water height $h(x, t)$, we consider $l_{1}, l_{2} \in[0,1]$ such that $l_{1}+l_{2}=1$, and we decompose the fluid along the vertical axis into two virtual layers of height

$$
h_{\alpha}=l_{\alpha} h, \alpha=1,2 .
$$

In what follows, $\alpha=1$ stands for the lower layer and $\alpha=2$ for the upper one (see Fig. 1). Let us denote

$$
\mathcal{L}_{1}(x, t)=\left\{z:-H(x, t) \leq z \leq z_{I}(x, t)\right\}, \mathcal{L}_{2}(x, t)=\left\{z: z_{I}(x, t) \leq z \leq \eta(x, t)\right\},
$$

where $z_{I}(x, t)=h_{1}-H . z_{\alpha}$ will denote the level of the middle point of the layers:

$$
z_{1}(x, t)=\frac{z_{I}-H}{2}, \quad z_{2}(x, t)=\frac{z_{I}+\eta}{2} .
$$

For a given function $(x, z, t) \rightarrow f(x, z, t)$, in the case that it is discontinuous at the interface $z_{I}$, let us denote

$$
f_{I^{+}}(x, t)=\lim _{\substack{z \rightarrow z_{I}(x, t) \\ z>z_{I}(x, t)}} f(x, z, t), \quad f_{I^{-}}(x, t)=\lim _{\substack{z \rightarrow z_{I}(x, t) \\ z<z_{I}(x, t)}} f(x, z, t),
$$


Fig. 1 Bilayer setting

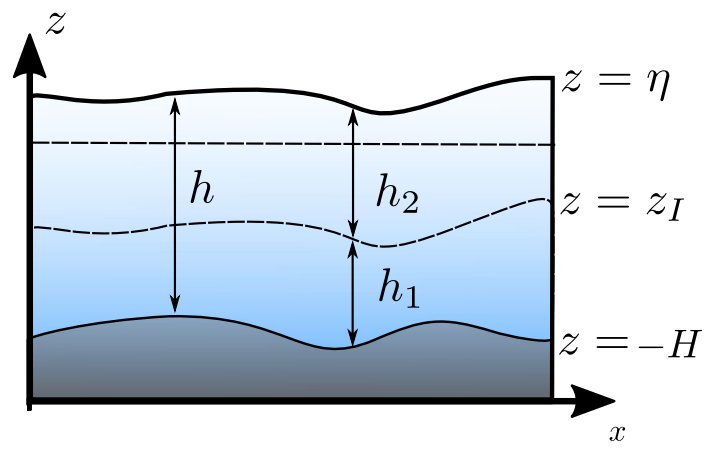

as well as $f_{b} \equiv f(x,-H(x, t), t), f_{\eta}(x, t) \equiv f(x, \eta(x, t), t)$.

As we mentioned before, to derive the model we assume a given vertical profile on the unknowns of the problem. First, a piecewise constant profile of the horizontal velocity is considered. We denote by $u_{1}(x, t)$ and $u_{2}(x, t)$ the horizontal velocities at layers $\mathcal{L}_{1}(x, t)$ and $\mathcal{L}_{2}(x, t)$, respectively. This is the main hypothesis for the derivation of the model, which corresponds to a shallow water regime. In fact, if we take $p=0$ in (4) and $l_{1}=1$, we can derive in the same way the SWE.

From previous assumption and the incompressibility condition (1a), we obtain that the vertical velocity has a piecewise linear profile. Let us recall that, using the notation introduced before, its limits at the interface level $z=z_{I}(x, t)$ are denoted by $w_{I^{ \pm}}$. The vertical velocity at the bottom and the free surface are denoted by $w_{b}$ and $w_{\eta}$, respectively.

Finally, we also consider a piecewise linear profile of the non-hydrostatic pressure, which can be determined from its atmospheric value, which is supposed to be zero, its limits at the interface and the pressure at the bottom. Thus we have $p_{\eta}=0$, and denote its limits at the interface level $z=z_{I}(x, t)$ by $p_{I^{ \pm}}$, and the pressure at the bottom, $p_{b}(x, t)$. We also denote by $p_{1}(x, t)$ and $p_{2}(x, t)$ the non-hydrostatic pressure part evaluated at the middle level of the corresponding layer, that is,

$$
p_{1}(x, t)=p\left(x, z_{1}(x, t), t\right), \quad p_{2}(x, t)=p\left(x, z_{2}(x, t), t\right) .
$$

From the incompressibility condition (1a), we get that a weak solution which is discontinuous at $z=z_{I}(x, t)$ must verify the following jump condition (see [34,35])

$$
w_{I^{+}}-w_{I^{-}}=\left(u_{2}-u_{1}\right) \partial_{x} z_{I} .
$$

Let us denote by $w_{1}$ and $w_{2}$ the vertical velocity at the middle level of the corresponding layer, that is,

$$
w_{1}(x, t)=w\left(x, z_{1}(x, t), t\right), \quad w_{2}(x, t)=w\left(x, z_{2}(x, t), t\right) .
$$

Then, the incompressibility condition (1a) inside each layer reads,

$$
\left\{\begin{array}{l}
\partial_{x} u_{1}+2 \frac{w_{1}-w_{b}}{h_{1}}=0, \\
\partial_{x} u_{2}+2 \frac{w_{2}-w_{I^{+}}}{h_{2}}=0 .
\end{array}\right.
$$


The integration over each layer of the incompressibility Eq. (1a), combined with conditions (2a), (3), gives the mass equations at each layer,

$$
\left\{\begin{array}{l}
\partial_{t} h_{1}+\partial_{x}\left(h_{1} u_{1}\right)=-\left(w_{I^{-}}-\left(\partial_{t} z_{I}+u_{1} \partial_{x} z_{I}\right)\right), \\
\partial_{t} h_{2}+\partial_{x}\left(h_{2} u_{2}\right)=\left(w_{I^{+}}-\left(\partial_{t} z_{I}+u_{2} \partial_{x} z_{I}\right)\right) .
\end{array}\right.
$$

Let us define

$$
\begin{aligned}
& \Gamma_{I^{-}}=w_{I^{-}}-\partial_{t} z_{I}-u_{1} \partial_{x} z_{I}, \\
& \Gamma_{I^{+}}=w_{I^{+}}-\partial_{t} z_{I}-u_{2} \partial_{x} z_{I} .
\end{aligned}
$$

Using the jump condition (6), we get $\Gamma_{I^{+}}=\Gamma_{I^{-}}$and we may define

$$
\Gamma_{I}=\frac{w_{I^{+}}+w_{I^{-}}}{2}-\partial_{t} z_{I}-\frac{u_{1}+u_{2}}{2} \partial_{x} z_{I} .
$$

Then Eq. (8) becomes

$$
\left\{\begin{array}{l}
\partial_{t} h_{1}+\partial_{x}\left(h_{1} u_{1}\right)=-\Gamma_{I}, \\
\partial_{t} h_{2}+\partial_{x}\left(h_{2} u_{2}\right)=\Gamma_{I} .
\end{array}\right.
$$

Thus, $\Gamma_{I}$ can be interpreted as an approximation of the mass transfer across the interface $z_{I}$. Moreover, by combining Eqs. (10a) and (10b) we obtain

$$
\Gamma_{I}=l_{1} \partial_{x}\left(h_{2} u_{2}\right)-l_{2} \partial_{x}\left(h_{1} u_{1}\right) .
$$

Following the procedure described in [34], and taking into account the piecewise linear discontinuous profile of non-hydrostatic pressure, we obtain the following horizontal momentum equations

$$
\begin{aligned}
& \partial_{x}\left(h_{1} u_{1}\right)+\partial_{x}\left(h_{1} u_{1}^{2}+h_{1} p_{1}\right)+\frac{u_{1}+u_{2}}{2} \Gamma_{I}-p_{b} \partial_{x} H-p_{I^{-}} \partial_{x} z_{I}=-g h_{1} \partial_{x} \eta . \\
& \partial_{x}\left(h_{2} u_{2}\right)+\partial_{x}\left(h_{2} u_{2}^{2}+h_{2} p_{2}\right)-\frac{u_{1}+u_{2}}{2} \Gamma_{I}+p_{I^{+}} \partial_{x} z_{I}=-g h_{2} \partial_{x} \eta .
\end{aligned}
$$

Note that the gradients of $p_{1}$ and $p_{2}$ appear because, inside each layer, we assume that the pressure is linear. The following vertical momentum equations are also deduced,

$$
\begin{aligned}
& \partial_{x}\left(h_{1} w_{1}\right)+\partial_{x}\left(h_{1} u_{1} w_{1}\right)+\frac{w_{1}+w_{2}}{2} \Gamma_{I}+p_{I^{-}}-p_{b}=0 . \\
& \partial_{x}\left(h_{2} w_{2}\right)+\partial_{x}\left(h_{2} u_{2} w_{2}\right)-\frac{w_{1}+w_{2}}{2} \Gamma_{I}-p_{I^{+}}=0 .
\end{aligned}
$$

We shall consider in this work the unknowns

$$
p_{b} \text { and } p_{I}:=p_{I^{-}},
$$

for the non-hydrostatic pressure unknowns. To close the system, we will suppose that

$$
p_{I^{+}}=\gamma_{1} p_{b}+\gamma_{2} p_{I}, \gamma_{1}+\gamma_{2} \neq 0,
$$

and therefore, due to the assumption on the non-hydrostatic vertical profile:

$$
p_{1}=\frac{p_{b}+p_{I}}{2}, p_{2}=\frac{\gamma_{1} p_{b}+\gamma_{2} p_{I}}{2} \text {. }
$$


The underlying reason to assume this discontinuity for the non-hydrostatic pressure is to introduce an artificial set of free parameters, which will help to improve the dispersive properties of the system (see Sect. 3).

Remark 1 Notice that for $\gamma_{1}=0, \gamma_{2}=1$ we recover the original system derived in [34], and for $\gamma_{1}=1, \gamma_{2}=\frac{\delta}{\delta-2}$ the approach

$$
p_{I^{+}}-p_{I^{-}}=\delta\left(p_{2}-p_{1}\right) .
$$

We will avoid the case $\gamma_{1}+\gamma_{2}=0$ (see Sect. 3.1 and "Appendix D.3").

To sum up, collecting all the equations described before, the system reads as:

$$
\left\{\begin{array}{l}
\partial_{t} h+\partial_{x}\left(l_{1} h u_{1}\right)+\partial_{x}\left(l_{2} h u_{2}\right)=0 \\
\partial_{t}\left(l_{1} h u_{1}\right)+\partial_{x}\left(l_{1} h u_{1}^{2}+l_{1} h p_{1}\right) \\
\quad+\frac{u_{1}+u_{2}}{2} \Gamma_{I}-p_{b} \partial_{x} H-p_{I} \partial_{x} z_{I}=-g l_{1} h \partial_{x} \eta \\
\partial_{t}\left(l_{1} h u_{2}\right)+\partial_{x}\left(l_{2} h u_{2}^{2}+l_{2} h p_{2}\right) \\
\quad-\frac{u_{1}+u_{2}}{2} \Gamma_{I}+\left(\gamma_{1} p_{b}+\gamma_{2} p_{I}\right) \partial_{x} z_{I}=-g l_{2} h \partial_{x} \eta \\
\partial_{t}\left(l_{1} h w_{1}\right)+\partial_{x}\left(l_{1} h u_{1} w_{1}\right)+\frac{w_{1}+w_{2}}{2} \Gamma_{I}=p_{b}-p_{I}, \\
\partial_{t}\left(l_{2} h w_{2}\right)+\partial_{x}\left(l_{2} h u_{2} w_{2}\right)-\frac{w_{1}+w_{2}}{2} \Gamma_{I}=\gamma_{1} p_{b}+\gamma_{2} p_{I} \\
\partial_{x} u_{1}+2 \frac{w_{1}-w_{b}}{l_{1} h}=0, \\
\partial_{x} u_{2}+2 \frac{w_{2}-w_{I}}{l_{2} h}=0,
\end{array}\right.
$$

where

$$
\left\{\begin{array}{l}
w_{I^{+}}=u_{2} \partial_{x} z_{I}-\partial_{x}\left(l_{1} h u_{1}\right)-\partial_{t} H, \\
w_{b}=-u_{1} \partial_{x} H-\partial_{t} H .
\end{array}\right.
$$

The system depends on the parameters $\left(l_{1}, \gamma_{1}, \gamma_{2}\right)$, which need to be chosen. This will be done following a criterion that improves the dispersive relations of the system. Note that the proposed system has the same number of variables as when considering a continuous pressure, that is $p_{I^{+}}-p_{I^{-}}=0$, that are

$$
h, u_{\alpha}, w_{\alpha}, p_{I}, p_{b}
$$


and therefore the computational cost of the improved model will be the same than the original one proposed in [34].

Finally, system (17) can be written in the compact form

$$
\left\{\begin{array}{l}
\partial_{t} \boldsymbol{U}+\partial_{x} \boldsymbol{F}(\boldsymbol{U})+\boldsymbol{B}(\boldsymbol{U}) \partial_{x} \boldsymbol{U}=\boldsymbol{G}(\boldsymbol{U}) \partial_{x} H+\mathcal{T}\left(h, \partial_{x} h, H, \partial_{x} H, \boldsymbol{P}, \partial_{x} \boldsymbol{P}\right), \\
\mathcal{B}\left(\boldsymbol{U}, \partial_{x} \boldsymbol{U}, H, \partial_{x} H\right)=\mathbf{0},
\end{array}\right.
$$

where we introduce the notation

$$
\boldsymbol{U}=\left(\begin{array}{c}
h \\
q_{u, 1} \\
q_{u, 2} \\
q_{w, 1} \\
q_{w, 2}
\end{array}\right), \boldsymbol{F}(\boldsymbol{U})=\left(\begin{array}{c}
l_{1} q_{u, 1}+l_{2} q_{u, 2} \\
\frac{q_{u, 1}^{2}}{h}+\frac{1}{2} g h^{2} \\
\frac{q_{u, 2}^{2}}{h}+\frac{1}{2} g h^{2} \\
\frac{q_{u, 1} q_{w, 1}}{h} \\
\frac{q_{u, 2} q_{w, 2}}{h}
\end{array}\right), \boldsymbol{G}(\boldsymbol{U})=\left(\begin{array}{c}
0 \\
g h \\
g h \\
0 \\
0
\end{array}\right),
$$

being

$$
q_{u, \alpha}=h u_{\alpha}, q_{w, \alpha}=h w_{\alpha}, \alpha=1,2
$$

the horizontal and vertical discharges.

$\boldsymbol{B}(\boldsymbol{U})$ is a matrix function such that $\boldsymbol{B}(\boldsymbol{U}) \partial_{x} \boldsymbol{U}$ involves the non-conservative products related to the mass transfer across interfaces that appear in the momentum equations

$$
\boldsymbol{B}(\boldsymbol{U})=\left(\begin{array}{ccccc}
0 & 0 & 0 & 0 & 0 \\
0 & -l_{2} \frac{u_{1}+u_{2}}{2} & l_{2} \frac{u_{1}+u_{2}}{2} & 0 & 0 \\
0 & l_{1} \frac{u_{1}+u_{2}}{2} & -l_{1} \frac{u_{1}+u_{2}}{2} & 0 & 0 \\
0 & -l_{2} \frac{w_{1}+w_{2}}{2} & l_{2} \frac{w_{1}+w_{2}}{2} & 0 & 0 \\
0 & l_{1} \frac{w_{1}+w_{2}}{2} & -l_{1} \frac{w_{1}+w_{2}}{2} & 0 & 0
\end{array}\right)
$$

Note that the corresponding equations for the horizontal and vertical momentum at the layer $\mathcal{L}_{\alpha}$, have been divided by $l_{\alpha}$. The limit case $l_{1}=1, l_{2}=0$ corresponds to the one-layer model presented in [16]. For the sake of simplicity, we will assume that $l_{\alpha} \in(0,1)$.

Finally, the non-hydrostatic terms are given by

$$
\boldsymbol{P}=\left(\begin{array}{c}
p_{b} \\
p_{I}
\end{array}\right), \mathcal{T}\left(h, \partial_{x} h, H, \partial_{x} H, \boldsymbol{P}, \partial_{x} \boldsymbol{P}\right)=-\left(\begin{array}{c}
0 \\
\partial_{x}\left(h p_{1}\right)-p_{b} \partial_{x} H / l_{1}-p_{I} \partial_{x} z_{I} / l_{1} \\
\partial_{x}\left(h p_{2}\right)+\left(\gamma_{1} p_{b}+\gamma_{2} p_{I}\right) \partial_{x} z_{I} / l_{2} \\
\left(p_{I}-p_{b}\right) / l_{1} \\
-\left(\gamma_{1} p_{b}+\gamma_{2} p_{I}\right) / l_{2}
\end{array}\right),
$$


where

$$
p_{1}=\frac{p_{b}+p_{I}}{2}, p_{2}=\frac{\gamma_{1} p_{b}+\gamma_{2} p_{I}}{2}
$$

and

$$
\mathcal{B}\left(\boldsymbol{U}, \partial_{x} \boldsymbol{U}, H, \partial_{x} H\right)=\left(\begin{array}{c}
l_{1} h \partial_{x} q_{u, 1}-2 q_{u, 1} \partial_{x} z_{1}+2 q_{w, 1}+2 h \partial_{t} H \\
2 l_{1} h \partial_{x} q_{u, 1}+l_{2} h \partial_{x} q_{u, 2}-2 q_{u, 2} \partial_{x} z_{2}+2 q_{w, 2}+2 h \partial_{t} H
\end{array}\right) .
$$

Operator (19) contains the incompressibility condition for each layer, and is obtained multiplying Eqs. (17f) and (17g) by $l_{1} h^{2}$ and $l_{2} h^{2}$ respectively. Equations (17h) and (17i) have been used as well. This allows to write the full two-layer system in terms of discharges.

Remark 2 Let us consider the underlying hydrostatic system given by

$$
\partial_{t} \boldsymbol{U}+\partial_{x} \boldsymbol{F}(\boldsymbol{U})+\boldsymbol{B}(\boldsymbol{U}) \partial_{x} \boldsymbol{U}=\boldsymbol{G}(\boldsymbol{U}) \partial_{x} H .
$$

Then the system (20) is hyperbolic for every $l_{1} \in(0,1)$. A proof is given in "Appendix A.1". In the case of $l_{1}=\frac{1}{2}$, the eigenvalues can be computed explicitly and are given by:

$$
\lambda_{1}=u_{1}, \lambda_{2}=u_{2}, \lambda_{3}=\frac{u_{1}+u_{2}}{2}, \lambda_{4,5}=\frac{u_{1}+u_{2}}{2} \pm \sqrt{g h+\frac{3}{4}\left(u_{1}-u_{2}\right)^{2}} .
$$

An approximation of the eigenvalues for the general case are given in "Appendix A.2".

Remark 3 Concerning the well-possessedness of the proposed system, if we restrict just to the hyperbolic problem (20), without non-hydrostatic pressure, some studies can be found although there is no final answer. For classical two-layer shallow water systems some results can be found in [54-57]. In the case of multi-layer shallow water systems, we may cite the work [7], where the authors prove that the multilayer model is hyperbolic for the case of two layers, but the question remains open for the general case. Another work related to this topic is [36]. In this paper, it is shown that the solution of the multilayer model is a particular weak solution of the Navier-Stokes system. Concerning multilayer problems with non hydrostatic pressure, to our knowledge, not much is known concerning well-possessedness.

\section{Linear Analysis of the New Model}

In this section, dispersive relations of the integrated two-layer system (17) are studied as usual (see $[25,45,46,52,62])$. In particular, we focus on the linear dispersion relation, the group velocity, and the linear shoaling. This constitutes a standard study of PDE systems for dispersive water waves modelling. The two first properties are related to the propagation of dispersive wave trains, and the latter with shoaling processes. This can occur when waves arrive at the continental shelf, from intermediate to shallow waters.

In Sect. 3.1, a flat bottom $H$ is assumed and the governing Eq. (17) are linearised around a steady state solution. Then, we conduct a standard Stokes-type Fourier analysis to obtain the linear dispersion relation and group velocity. In Sect. 3.2, a shoaling analysis of the linearised equations is carried out. The linear dispersion relation, group velocity and linear shoaling are derived following the procedure described in [62]. Remark that the studied dispersive relations are dependent on the parameters $\left(l_{1}, \gamma_{1}, \gamma_{2}\right)$. In Sect. 3.3 the aforementioned $\left(l_{1}, \gamma_{1}, \gamma_{2}\right)$ parameters will be chosen through examination of the derived dispersive relations following the standard procedure on such topics (see $[46,52,62])$. 


\subsection{Linear Dispersion Relation}

Assuming that both $\eta$ and $u$ are very small perturbation of a still steady state, and flat bathymetry, the linearised version of system (17) reads

$$
\left\{\begin{array}{l}
\partial_{t} \eta+H \partial_{x}(\bar{u})=0, \\
\partial_{t} U+g \partial_{x} \eta I_{2}+\frac{1}{2} A_{1} \partial_{x} P=0, \\
H \partial_{t} W+A_{2} P=0, \\
H A_{3} \partial_{x} U+2 W=0,
\end{array}\right.
$$

being

$$
\begin{aligned}
U & =\left(\begin{array}{l}
u_{1} \\
u_{2}
\end{array}\right), W=\left(\begin{array}{l}
w_{1} \\
w_{2}
\end{array}\right), P=\left(\begin{array}{l}
p_{b} \\
p_{I}
\end{array}\right), \\
I_{2} & =\left(\begin{array}{ll}
1 & 0 \\
0 & 1
\end{array}\right), A_{1}=\left(\begin{array}{cc}
1 & 1 \\
\gamma_{1} & \gamma_{2}
\end{array}\right), A_{2}=\left(\begin{array}{cc}
-1 / l_{1} & 1 / l_{1} \\
-\gamma_{1} / l_{2} & -\gamma_{2} / l_{2}
\end{array}\right), A_{3}=\left(\begin{array}{cc}
l_{1} & 0 \\
2 l_{1} & l_{2}
\end{array}\right) .
\end{aligned}
$$

If we assume $\gamma_{1}+\gamma_{2} \neq 0$, then after some algebraic manipulations, the system (21) can be expressed in terms of $\eta, U$ and its derivatives,

$$
\left\{\begin{array}{l}
\partial_{t} \eta+H \partial_{x}(\bar{u})=0, \\
\partial_{t} U+g \partial_{x} \eta I_{2}+\frac{1}{4} H^{2} A_{1} A_{2}^{-1} A_{3} \partial_{x x t} U=0 .
\end{array}\right.
$$

We shall make a Stokes-type Fourier analysis and look for first-order solutions of the form,

$$
\eta(x, t)=\eta_{0} e^{i(\omega t-k x)}, U(x, t)=\left(\begin{array}{l}
u_{1} \\
u_{2}
\end{array}\right) e^{i(\omega t-k x)},
$$

where $\eta_{0}$ is the wave amplitude, $\omega$ is the cyclic frequency and $k$ the wave number. By substituting (23) into (22), we get the linear system

$$
\left(\begin{array}{c|c}
\Lambda & g \\
l_{1} H l_{2} H & -C
\end{array}\right)\left(\begin{array}{l}
u_{1} \\
u_{2} \\
\eta_{0}
\end{array}\right)=\left(\begin{array}{l}
0 \\
0 \\
0
\end{array}\right)
$$

where $C$ is the wave celerity defined by $C=\omega / k$ and

$$
\Lambda=-C\left(I_{2}+\frac{1}{4}(k H)^{2} A_{1} A_{2}^{-1} A_{3}\right) .
$$

Looking for non-trivial solutions, the matrix of the linear system (24) must be singular, and yields the linear dispersion relation

$$
\frac{C^{2}}{g H}=\frac{N_{0}+N_{1}(k H)^{2}}{D_{0}+D_{1}(k H)^{2}+D_{2}(k H)^{4}} .
$$


Since $l_{2}=1-l_{1}$, the coefficients $N_{0}, N_{1}, D_{0}, D_{1}, D_{2}$, given in "Appendix B.1", are solely functions of $l_{1}, \gamma_{1}, \gamma_{2}$.

Now, we can derive the group velocity $C_{g}$, which is essentially computed by taking the derivative of $C$ in (25) with respect to the wave number $k$. This will be related with the propagation of irregular wave trains (see [52]) and it is defined as

$$
C_{g}=C+k \partial_{k} C
$$

Remark 4 If the relation $\gamma_{1}+\gamma_{2}=0$ holds, then regarding the linear vertical velocity equation in (21), one has that $P$ can not be uniquely expressed in terms of $\partial_{t} W$, due to the fact that $A_{2}$ is a singular matrix. According to this, we assume in this work that $\gamma_{1}+\gamma_{2} \neq 0$, and thus the matrix $A_{2}$ has an inverse $A_{2}^{-1}$.

\subsection{Linear Shoaling Analysis}

Madsen and Sørensen introduced the linear shoaling gradient as another quantity to measure the applicability of Boussinesq equations (see [52]). The linear shoaling of the present twolayer model can be expressed as,

$$
\frac{\partial_{x} \eta}{\eta}=-\frac{\partial_{x} H}{H} \gamma
$$

where the shoaling gradient $\gamma$ is a function of the wave number $k$ and $H$ and it is determined using the concept of the constancy of the energy flux,

$$
\partial_{x}\left(\eta^{2} C_{g}\right)=0,
$$

and thus,

$$
\frac{\partial_{x} \eta}{\eta}=-\frac{1}{2} \frac{\partial_{x} C_{g}}{C_{g}} .
$$

Given the group velocity, it can be seen

$$
\frac{\partial_{x} C_{g}}{C_{g}}=S_{1} \frac{\partial_{x}(k H)}{k H}
$$

where $S_{1}$ is a function to be determined such that the equality holds. Taking the derivative of the dispersive relation with respect to $x$, gives

$$
\frac{\partial_{x} k}{k}=S_{2} \frac{\partial_{x} H}{H}
$$

where, again, $S_{2}$ is another function to be determined. Finally, the linear shoaling gradient is given by:

$$
\gamma=\frac{1}{2} S_{1}\left(1+S_{2}\right)
$$

The determination of $\gamma$ is performed on a computer using symbolic calculation. The resulting expression is tedious and will not be given here. 


\subsection{Dispersive Optimization}

For the studied quantities, i.e. wave celerity, group velocity and linear shoaling, a reference formula can be derived from the Stokes linear theory. The exact linear dispersion relation is given by

$$
\frac{C_{e}^{2}}{g H}=\frac{\tanh (k H)}{k H},
$$

and group velocity, $C_{g, e}$, can be derived from the above expression. Finally, based on Stokes linear theory Madsen \& Sørensen derived the exact shoaling gradient (see [52]):

$$
\gamma_{e}=k H \tanh (k H) \frac{(1-k H \tanh (k H))\left(1-\tanh ^{2}(k H)\right)}{\left(\tanh (k H)+k H\left(1-\tanh ^{2}(k H)\right)\right)^{2}} .
$$

Now, the most accurate set of parameters $l_{1}, \gamma_{1}$ and $\gamma_{2}$, in a sense that will be described later, will be chosen in this section attending to the previous formulas. For this analysis, a representation of the overall error, including errors in wave celerity, group velocity and shoaling, is sought. To do that, we shall minimize the error

$$
\Delta_{s}\left(l_{1}, \gamma_{1}, \gamma_{2}\right)=\int_{0}^{s} \frac{1}{k H}\left(\frac{\left|C-C_{e}\right|}{\left|C_{e}\right|}+\frac{\left|C_{g}-C_{g, e}\right|}{\left|C_{g, e}\right|}+\frac{\left|\gamma-\gamma_{e}\right|}{\left|\gamma_{e}\right|}\right) d k H
$$

and therefore we define

$$
\left(l_{1}^{(s)}, \gamma_{1}^{(s)}, \gamma_{2}^{(s)}\right):=\arg \min _{\substack{\left(l_{1}^{*}, \gamma_{1}^{*}, \gamma_{2}^{*}\right) \\ l_{1}^{*} \in(0,1) \\ \gamma_{1}+\gamma_{2} \neq 0}}\left[\Delta_{s}\left(l_{1}^{*}, \gamma_{1}^{*}, \gamma_{2}^{*}\right)\right]
$$

When simulating dispersive water waves, errors at low wave numbers $k$ are more relevant than errors at high wave numbers. Due to that, as in [47], the sum of the relative errors is divided by $k H$ inside the integral (26). Then, the proposed election of $\left(l_{1}^{(s)}, \gamma_{1}^{(s)}, \gamma_{2}^{(s)}\right)$ minimizes properly the sum of the relative errors of wave celerity, group velocity and linear shoaling, with respect to the reference formulas in a range of $k H \in[0, s]$. The integral is approximated numerically via Gaussian quadrature points, avoiding the singularity at $k H=0$. The $\arg \min$ function is approximated by using an iterative method for non-linear optimization.

Attending to the range of applicability, two set of parameters are obtained. The first one (see Fig. 2) is

$$
\left(l_{1}^{(5)}, \gamma_{1}^{(5)}, \gamma_{2}^{(5)}\right)=(0.4929,-0.1530,1.1192)
$$

This choice, leads to an excellent agreement with Stokes first order theory for $k H$ up to 5 . The percentage errors in phase celerity is less than $0.8 \%$ and for the group velocity is less than $2 \%$. Linear shoaling is reproduced very well in this range also. Another choice (see Fig. 3) for an extended range is

$$
\left(l_{1}^{(15)}, \gamma_{1}^{(15)}, \gamma_{2}^{(15)}\right)=(0.7194,0.1386,0.7305) .
$$

This ensures a percentage error for the celerity less than $1.5 \%$ for $k H$ up to 15 . For the group velocity this choice provides an error less than $8 \%$ for $k H$ up to 10 . Figures 2 and 3, also show a comparison with the standard two-layer approximation corresponding to the choice:

$$
\left(l_{1}^{(2 L)}, \gamma_{1}^{(2 L)}, \gamma_{2}^{(2 L)}\right)=(1 / 2,0,1)
$$



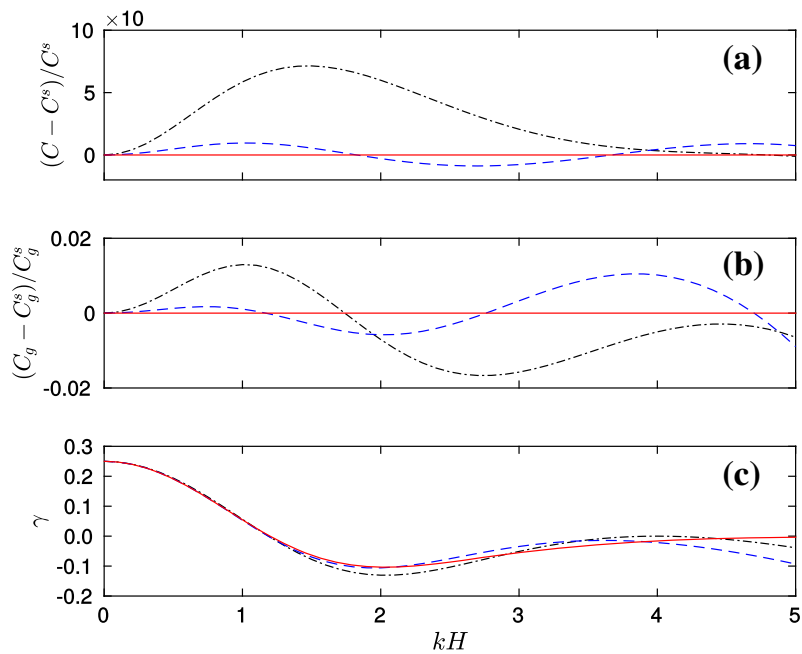

Fig. 2 a, b Relative errors for the phase and group velocity respectively. $\mathbf{c}$ The comparisons with the reference shoaling gradient (in red). The results with the optimized parameters $\left(l_{1}^{(5)}, \gamma_{1}^{(5)}, \gamma_{2}^{(5)}\right)$ are given in blue dashed lines and with the non-optimized parameters $\left(l_{1}^{(2 L)}, \gamma_{1}^{(2 L)}, \gamma_{2}^{(2 L)}\right)$ in black dash-dotted lines (Color figure online)
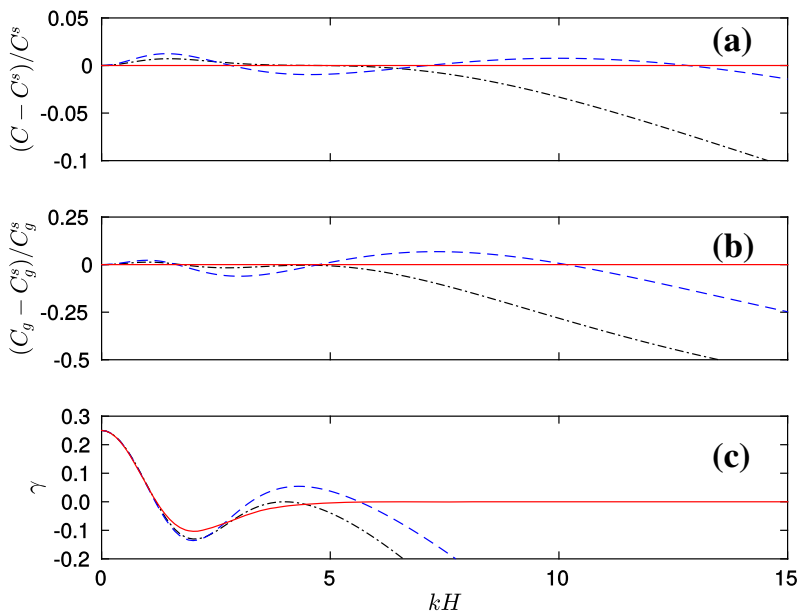

Fig. 3 a, b Relative errors for the phase and group velocity respectively. $\mathbf{c}$ The comparisons with the reference shoaling gradient (in red). The results with the optimized parameters $\left(l_{1}^{(15)}, \gamma_{1}^{(15)}, \gamma_{2}^{(15)}\right)$ are given in blue dashed lines and with the non-optimized parameters $\left(l_{1}^{(2 L)}, \gamma_{1}^{(2 L)}, \gamma_{2}^{(2 L)}\right)$ in black dash-dotted lines (Color figure online)

Although the second optimization (28) does not seem to improve the errors with respect to (29), it ensures reasonable errors for an extended range of $k H$.

Let us remark that the model proposed in this work presents a good agreement for linear shoaling gradient with just two layers. In order to have similar results, at least five layers are needed for multi-layer models like the one presented in [11]. Moreover, the results for the phase velocity with parameters $\left(l_{1}^{(15)}, \gamma_{1}^{(15)}, \gamma_{2}^{(15)}\right)$ show that we obtain a smaller relative 
error for $k H$ up to 15 , when compared with the two-layer system proposed in [27]. This means that the model proposed here can achieve better dispersive properties than models that have similar o even more computational complexity.

As the equations are largely used to simulate shallow water flows, the linear analysis is supplemented with an asymptotic analysis in the limit $k H \rightarrow 0$. To do that, we compare the resulting Taylor expansion of the phase velocity (25) with the one coming from the Stokes linear theory at $\operatorname{order} \mathcal{O}(k H)^{4}$ :

$$
\begin{aligned}
\frac{C_{e}^{2}}{g H} & =1-\frac{1}{3}(k H)^{2}+\mathcal{O}(k H)^{4}, \\
\frac{C^{2}}{g H} & =1-\frac{\gamma_{1}+\gamma_{2}-l_{1}\left(\gamma_{1}+\gamma_{2}+l_{1}\left(\gamma_{1}+\gamma_{2}-2\left(\gamma_{2}-1\right) l_{1}\right)-2\right)}{4\left(\gamma_{1}+\gamma_{2}\right)}(k H)^{2}+\mathcal{O}(k H)^{4},
\end{aligned}
$$

which coincides for

$$
\gamma_{1}=\frac{3 l_{1}\left(1-l_{1}\right)\left(2-\gamma_{2}-2\left(\gamma_{2}-1\right) l_{1}\right)-\gamma_{2}}{3 l_{1}\left(l_{1}+1\right)+1} .
$$

Note that the original two-layer non-hydrostatic system derived in [34], which corresponds to the election of $l_{1}=\frac{1}{2}$ and $\gamma_{1}=0, \gamma_{2}=1$, verify at $\operatorname{order} \mathcal{O}(k H)^{4}$ :

$$
\frac{C_{2 L}^{2}}{g H}=1-\frac{5}{16}(k H)^{2}+\mathcal{O}(k H)^{4} .
$$

Thus, the proposed system can satisfy up to $\operatorname{order} \mathcal{O}(k H)^{4}$ the Stokes linear theory, by setting $\gamma_{1}$ as in (30). The parameters $l_{1}$ and $\gamma_{2}$ are still free parameters, and a similar tuning as the one explained in this subsection can be made in order improve the linear dispersive relation for higher values of $k H$.

\section{Numerical Scheme}

We describe now the numerical scheme used to discretize the system (18) for a fixed bed, that is, $\partial_{t} H=0$. The numerical scheme employed is based on a two-step projection-correction method. First, we shall solve the underlying non-conservative hydrostatic and hyperbolic system given by

$$
\partial_{t} \boldsymbol{U}+\partial_{x} \boldsymbol{F}(\boldsymbol{U})+\boldsymbol{B}(\boldsymbol{U}) \partial_{x} \boldsymbol{U}=\boldsymbol{G}(\boldsymbol{U}) \partial_{x} H .
$$

Then, in a second step, non-hydrostatic terms will be taken into account. System (31) is discretized by a second order finite volume Polynomial Viscosity Matrix (hereinafter PVM) positive-preserving well-balanced segment path-conservative method (see [17]). As usual, we consider a set of $N$ finite volume cells $I_{i}=\left[x_{i-1 / 2}, x_{i+1 / 2}\right]$ with constant lengths $\Delta x$ and define

$$
\boldsymbol{U}_{i}(t)=\frac{1}{\Delta x} \int_{I_{i}} \boldsymbol{U}(x, t) d x,
$$

the cell average of the function $\boldsymbol{U}(x, t)$ on cell $I_{i}$ at time $t$. Regarding non-hydrostatic terms, we consider the mid-points $x_{i}$ of each cell $I_{i}$ and denote the point values of the function $\boldsymbol{P}$ at time $t$ by

$$
\boldsymbol{P}_{i}(t)=\boldsymbol{P}\left(x_{i}, t\right) .
$$

Non-hydrostatic terms will be approximated by second order compact finite-differences. 


\subsection{Time Stepping}

Assume given time steps $\Delta t^{n}$, and denote $t^{n}=\sum_{j \leq n} \Delta t^{j}$. To obtain second order accuracy in time, the two-stage second-order TVD Runge-Kutta scheme [39] is adopted. At the $k$ th stage, $k \in\{1,2\}$, the two-step projection-correction method is given by

$$
\left\{\begin{array}{l}
\frac{\boldsymbol{U}^{(\widetilde{k})}-\boldsymbol{U}^{(k-1)}}{\Delta t}+\partial_{x} \boldsymbol{F}\left(\boldsymbol{U}^{(k-1)}\right)+\boldsymbol{B}\left(\boldsymbol{U}^{(k-1)}\right) \partial_{x} \boldsymbol{U}^{(k-1)}=\boldsymbol{G}\left(\boldsymbol{U}^{(k-1)}\right) \partial_{x} H \\
\frac{\boldsymbol{U}^{(k)}-\boldsymbol{U}^{(\widetilde{k})}}{\Delta t}=\mathcal{T}\left(h^{(k)}, \partial_{x} h^{(k)}, H, \partial_{x} H, \boldsymbol{P}^{(k)}, \partial_{x} \boldsymbol{P}^{(k)}\right) \\
\mathcal{B}\left(\boldsymbol{U}^{(k)}, \partial_{x} \boldsymbol{U}^{(k)}, H, \partial_{x} H\right)=\mathbf{0}
\end{array}\right.
$$

where $\boldsymbol{U}^{(0)}$ is $\boldsymbol{U}$ at the time level $t^{n}, \boldsymbol{U}^{(\widetilde{k})}$ is an intermediate value in the two-step projectioncorrection method that contains the numerical solution of the SWE [system (32a)] at the corresponding $k$ th stage of the Runge-Kutta, and $\boldsymbol{U}^{(k)}$ is the $k$ th stage estimate. After that, a final value of the solution at the $t^{n+1}$ time level is obtained:

$$
\boldsymbol{U}^{n+1}=\frac{1}{2} \boldsymbol{U}^{n}+\frac{1}{2} \boldsymbol{U}^{(2)} .
$$

Note that, Eqs. (32b-32c) requires, at each stage of the calculation respectively, to solve a Poisson-like equation for each one of the variables contained in $\boldsymbol{P}^{(k)}$. This will be described below.

Remark that the usual CFL restriction should be considered for the computation of the time step $\Delta t$.

\subsection{Finite Volume Discretization for the Underlying Hyperbolic System}

A second order path-conservative PVM scheme for the discretization in space of the system (32a), at the $k$ th stage of the Runge-Kutta, reads as follows (see [17,19] for more details about PVM schemes and the extension to high order):

$$
\frac{\boldsymbol{U}^{(\widetilde{k})}-\boldsymbol{U}^{(k-1)}}{\Delta t}=-\frac{1}{\Delta x}\left(D_{i+1 / 2}^{(k-1),-}+D_{i-1 / 2}^{(k-1),+}+\mathcal{I}_{i}\right),
$$

where, skipping the time dependence in $k$ to relax the notation,

$$
\begin{aligned}
D_{i+1 / 2}^{-}= & D_{i+1 / 2}^{-}\left(\boldsymbol{U}_{i+1 / 2}^{-}, \boldsymbol{U}_{i+1 / 2}^{+}, H_{i+1 / 2}^{-}, H_{i+1 / 2}^{+}\right) \\
= & \frac{1}{2}\left(\boldsymbol{F}\left(\boldsymbol{U}_{i+1 / 2}^{+}\right)-\boldsymbol{F}\left(\boldsymbol{U}_{i+1 / 2}^{-}\right)+\boldsymbol{B}\left(\tilde{\boldsymbol{U}}_{i+1 / 2}\right)\left(\boldsymbol{U}_{i+1 / 2}^{+}-\boldsymbol{U}_{i+1 / 2}^{-}\right)\right. \\
& \left.-\boldsymbol{G}_{i+1 / 2}\left(H_{i+1 / 2}^{+}-H_{i+1 / 2}^{-}\right)\right) \\
& \pm \frac{1}{2} Q_{i+1 / 2}\left(\left(\boldsymbol{U}_{i+1 / 2}^{+}-\boldsymbol{U}_{i+1 / 2}^{-}\right)-A_{i+1 / 2}^{-1} \boldsymbol{G}_{i+1 / 2}\left(H_{i+1 / 2}^{+}-H_{i+1 / 2}^{-}\right)\right), \\
\mathcal{I}_{i}= & \boldsymbol{F}\left(\boldsymbol{U}_{i+1 / 2}^{-}\right)-\boldsymbol{F}\left(\boldsymbol{U}_{i-1 / 2}^{+}\right)+\boldsymbol{B}\left(\boldsymbol{U}_{i}\right)\left(\boldsymbol{U}_{i+1 / 2}^{-}-\boldsymbol{U}_{i-1 / 2}^{+}\right) \\
& -\boldsymbol{G}\left(\boldsymbol{U}_{i}\right)\left(H_{i+1 / 2}^{-}-H_{i-1 / 2}^{+}\right)
\end{aligned}
$$


where

$$
\tilde{\boldsymbol{U}}_{i+1 / 2}=\left(\begin{array}{c}
\tilde{h}_{i+1 / 2} \\
\widetilde{h}_{i+1 / 2} \widetilde{u}_{1, i+1 / 2} \\
\tilde{h}_{i+1 / 2} \widetilde{u}_{2, i+1 / 2} \\
\tilde{h}_{i+1 / 2} \widetilde{w}_{1, i+1 / 2} \\
\tilde{h}_{i+1 / 2} \widetilde{w}_{2, i+1 / 2}
\end{array}\right), \boldsymbol{G}_{i+1 / 2}=\left(\begin{array}{c}
0 \\
g \widetilde{h}_{i+1 / 2} \\
g \widetilde{h}_{i+1 / 2} \\
0 \\
0
\end{array}\right),
$$

$\tilde{h}_{i+1 / 2}=\frac{h_{i+1 / 2}^{+}+h_{i+1 / 2}^{-}}{2}, \tilde{u}_{\alpha, i+1 / 2}=\frac{u_{\alpha, i+1 / 2}^{+} \sqrt{h_{i+1 / 2}^{+}}+u_{\alpha, i+1 / 2}^{-} \sqrt{h_{i+1 / 2}^{-}}}{\sqrt{h_{i+1 / 2}^{+}}+\sqrt{h_{i+1 / 2}^{-}}}$for $\alpha=1,2$.

$\widetilde{w}_{\alpha, i+1 / 2}$ is defined in the same way as $\tilde{u}_{\alpha, i+1 / 2}$. The vector $\boldsymbol{U}_{i+1 / 2}^{ \pm}$is defined by a reconstruction procedure on the variables to the to the left $\left(^{-}\right)$and right $\left(^{+}\right)$of the inter-cell $x_{i+1 / 2}$ : $h_{i+1 / 2}^{ \pm}, \eta_{i+1 / 2}^{ \pm}, u_{\alpha, i+1 / 2}^{ \pm}, w_{\alpha, i+1 / 2}^{ \pm}$. This reconstruction procedure is done using a MUSCL reconstruction operator (see [66]), combined with a minmod limiter. The MUSCL reconstruction operator takes into account the positivity of the water height. Finally, the variable $H_{i+1 / 2}^{ \pm}$is recovered from $H_{i+1 / 2}^{ \pm}=h_{i+1 / 2}^{ \pm}-\eta_{i+1 / 2}^{ \pm}$. This procedure allows the scheme to deal with emerging topographies, since the variable $\eta$ is reconstructed instead oh $H$ in those situations, which means that the gradient of $\eta$ is set to zero to avoid spurious non-physical pressure forces (see [18]).

$Q_{i+1 / 2}$ is the viscosity matrix associated to the numerical method. For PVM schemes, $Q_{i+1 / 2}$ is obtained by a polynomial evaluation of the Roe Matrix. In this work, $Q_{i+1 / 2}$ is defined as

$$
Q_{i+1 / 2}=\beta_{0} I d+\beta_{1} A_{i+1 / 2},
$$

being

$$
\beta_{0}=\frac{S_{R}\left|S_{L}\right|-S_{L}\left|S_{R}\right|}{S_{R}-S_{L}}, \quad \beta_{1}=\frac{\left|S_{R}\right|-\left|S_{L}\right|}{S_{R}-S_{L}},
$$

where $S_{L}, S_{R}$ are estimates of the smallest and biggest wave speeds respectively at the interface $x_{i+1 / 2}$, as it is usually considered for such methods. Here, we use

$$
\begin{aligned}
& S_{L}=\min \left(\hat{u}_{i+1 / 2}-\sqrt{g \hat{h}_{i+1 / 2}}, \bar{u}_{i}-\sqrt{g h_{i}}\right), \\
& S_{R}=\max \left(\hat{u}_{i+1 / 2}+\sqrt{g \hat{h}_{i+1 / 2}}, \bar{u}_{i+1}+\sqrt{g h_{i+1}}\right),
\end{aligned}
$$

where

$$
\hat{u}_{i+1 / 2}=\frac{\bar{u}_{i} \sqrt{h_{i}}+\bar{u}_{i+1} \sqrt{h_{i+1}}}{\sqrt{h_{i}}+\sqrt{h_{i+1}}}, \bar{u}_{i}=l_{1} u_{1, i}+l_{2} u_{2, i} .
$$

$A_{i+1 / 2}$ denotes the matrix

$$
A_{i+1 / 2}=\mathcal{J}_{\mathcal{F}}\left(\tilde{\boldsymbol{U}}_{i+1 / 2}\right)+\boldsymbol{B}\left(\tilde{\boldsymbol{U}}_{i+1 / 2}\right),
$$

being $\mathcal{J}_{F}$ the Jacobian matrix of the flux $\boldsymbol{F}$. 
The scheme is a segment path-conservative extension of HLL scheme [41] for nonconservative systems. Notice that the term

$$
Q_{i+1 / 2} A_{i+1 / 2}{ }^{-1} \boldsymbol{G}_{i+1 / 2}\left(H_{i+1 / 2}^{+}-H_{i+1 / 2}^{-}\right)
$$

in (35) can be interpreted as the up-winding part of the source term discretization. This term makes no sense if any of the eigenvalues of $A_{i+1 / 2}$ vanishes. One way to deal with this kind of resonant problems has been proposed in [20].

Remark 5 It is clear that the concept of weak solution and the definition of path-conservative schemes strongly depends on the chosen family of paths, which is a priori arbitrary. The crucial question is thus how to choose the "good" family of paths. The answer to this question is not easy and it is not the scope of this article. We refer to [21] for a detailed discussion on the subject. In this paper, we consider the canonical path that is the segment path. In the reference [13] a negative result of convergence for path-conservative numerical methods was given together with several numerical examples. Later, a new example was given in [2]. These results have led in many cases to an over-simplified picture according to which these convergence difficulties are a specific drawback of path-conservative methods.

Moreover, the experience proves that path-conservative numerical methods and in particular PVM schemes converge with the expected order of accuracy and, under the adequate CFL condition, with the same stability property as their conservative counterparts for geophysical problems. The difficulty comes from the fact that the limits of numerical solutions may differ from the correct ones in the case of non-conservative systems as weak solutions of non-conservative systems may be defined in infinitely many ways (paths).

In fact, the key point to understand the problem of convergence in non-conservative systems is the following: the limits of the numerical solutions satisfy a jump condition which is related to the numerical viscosity of the method and not to the physically relevant one. Of course, this phenomenon affects any numerical method in which the small-scale effects (the vanishing diffusion and/or dispersion) are not taken into account, regardless of whether it is path-conservative or not. For instance, even the Godunov method based on the right weak solutions of the Riemann problems fails, in general, to converge to the right solution: this is due to the numerical viscosity introduced in the averaging step.

Remark 6 The numerical scheme considered for the underlying hyperbolic system (32a and 34) is well-balanced for water at rest solutions and linearly $L^{\infty}-$ stable under the usual CFL condition. Moreover, the scheme is positive preserving for the water height for a smooth bathymetry under $\frac{1}{2} C F L$ condition.

\subsection{Finite Difference Discretization for the Non-hydrostatic Terms}

In this subsection we describe the discretization of the non-hydrostatic terms. From Eq. (32b), for every $k \in\{1,2\}$ of the Runge-Kutta stage, we get 


$$
\left\{\begin{array}{l}
q_{u, 1}^{(k)}=q_{u, 1}^{(\widetilde{k})}-\Delta t\left(\partial_{x}\left(h^{(k)} p_{1}^{(k)}\right)-\frac{1}{l_{1}}\left(p_{b}^{(k)} \partial_{x} H+p_{I}^{(k)} \partial_{x} z I^{(k)}\right)\right) \\
q_{u, 2}^{(k)}=q_{u, 2}^{(\widetilde{k})}-\Delta t\left(\partial_{x}\left(h^{(k)} p_{2}^{(k)}\right)+\frac{1}{l_{2}}\left(\gamma_{1} p_{b}^{(k)}+\gamma_{2} p_{I}^{(k)}\right) \partial_{x} z I^{(k)}\right) \\
q_{w, 1}^{(k)}=q_{w, 1}^{(\widetilde{k})}-\frac{\Delta t}{l_{1}}\left(p_{b}^{(k)}-p_{I}^{(k)}\right) \\
q_{w, 2}^{(k)}=q_{w, 2}^{(\widetilde{k})}-\frac{\Delta t}{l_{2}}\left(\gamma_{1} p_{I}^{(k)}+\gamma_{2} p_{b}^{(k)}\right) .
\end{array}\right.
$$

Replacing Eqs. (36a-36d) into (32c), we obtain the Poisson-like equations

$$
\begin{aligned}
& a_{1} \partial_{x x} p_{b}^{(k)}+a_{2} \partial_{x} p_{b}^{(k)}+a_{3} p_{b}^{(k)}+a_{4} \partial_{x x} p_{I}^{(k)}+a_{5} \partial_{x} p_{I}^{(k)}+a_{6} p_{I}^{(k)}=R H S_{1}, \\
& b_{1} \partial_{x x} p_{b}^{(k)}+b_{2} \partial_{x} p_{b}^{(k)}+b_{3} p_{b}^{(k)}+b_{4} \partial_{x x} p_{I}^{(k)}+b_{5} \partial_{x} p_{I}^{(k)}+b_{6} p_{I}^{(k)}=R H S_{2}
\end{aligned}
$$

where the coefficients $a_{j}, b_{j}$ as well as the Right-Hand-Sides $R H S_{j}, j \in\{1,2\}$, are given in "Appendix D.1". We point out the dependency of the aforementioned coefficients

$$
a_{j} \equiv a_{j}\left(\boldsymbol{U}^{(k)}\right), b_{j} \equiv b_{j}\left(\boldsymbol{U}^{(k)}\right), R H S_{j} \equiv R H S_{j}\left(\boldsymbol{U}^{(\widetilde{k})}\right)
$$

and remark that, due to the form of the numerical scheme and the operator $\mathcal{T}$, then one has that $h^{(k)}=h^{(\widetilde{k})}$, and thus

$$
a_{j} \equiv a_{j}\left(\boldsymbol{U}^{(\widetilde{k})}\right), b_{j} \equiv b_{j}\left(\boldsymbol{U}^{(\widetilde{k})}\right), \operatorname{RHS}_{j} \equiv \operatorname{RH} S_{j}\left(\boldsymbol{U}^{(\widetilde{k})}\right) .
$$

Equations (37) and (38) are discretized using second order finite differences. In order to obtain point value approximations of the non-hydrostatic pressure variables, terms $p_{I, i}^{(k)}, p_{b, i}^{(k)}$ and $R H S_{j, i}, j \in\{1,2\}, i \in\{1, \ldots, N\}$ will be approximated at every mid point $x_{i}$ of each cell $I_{i}$. In the same way, the corresponding space derivatives will be approximated using compact second order finite differences

$$
\partial_{x} \boldsymbol{P}_{i}^{(k)}=\frac{\boldsymbol{P}_{i+1}^{(k)}-\boldsymbol{P}_{i-1}^{(k)}}{2 \Delta x}, \partial_{x x} \boldsymbol{P}_{i}^{(k)}=\frac{\boldsymbol{P}_{i+1}^{(k)}-2 \boldsymbol{P}_{i}^{(k)}+\boldsymbol{P}_{i-1}^{(k)}}{\Delta x^{2}} .
$$

To compute the coefficients $a_{j}, b_{j}, R H S_{j}$, that contains terms depending on $\boldsymbol{U}^{(\widetilde{k})}$ as well as first derivatives $\partial_{x} \boldsymbol{U}^{(\widetilde{k})}$, we will use the averaged values on the cell $I_{i}$ of variables $\boldsymbol{U}^{(\widetilde{k})}$ and $H$ as second order point value approximations of $\boldsymbol{U}_{i}^{(\widetilde{k})}$ and $H_{i}$ at the centre of the cell $I_{i}$. The space derivatives are computed from the averaged values using compact second order finite differences

$$
\partial_{x} \boldsymbol{U}_{i}^{(\widetilde{k})}=\frac{\boldsymbol{U}_{i+1}^{(\widetilde{k})}-\boldsymbol{U}_{i-1}^{(\widetilde{k})}}{2 \Delta x}, \partial_{x} H_{i}=\frac{H_{i+1}-H_{i-1}}{2 \Delta x} .
$$

After replacing (39) and (40) in (37) and (38), one has to solve a linear system

$$
A \mathcal{P}=\mathcal{R H} \mathcal{S}
$$


where

$$
\mathcal{R} \mathcal{H} \mathcal{S}=\left(\begin{array}{c}
\mathcal{R} \mathcal{H} \mathcal{S}_{1} \\
\mathcal{R H}_{2}
\end{array}\right), \mathcal{R} \mathcal{H} \mathcal{S}_{j}=\left(\begin{array}{c}
R H S j, 1 \\
\vdots \\
R H S j, N
\end{array}\right), \mathcal{P}=\left(\begin{array}{c}
p_{b, 1}^{(k)} \\
\vdots \\
p_{b, N}^{(k)} \\
p_{I, 1}^{(k)} \\
\vdots \\
p_{I, N}^{(k)}
\end{array}\right),
$$

and

$$
\boldsymbol{A}=\boldsymbol{D}+\boldsymbol{M}, \boldsymbol{D}=\left(\begin{array}{c|c}
T_{(1)} & \mathbf{0} \\
\hline \mathbf{0} & T_{(2)}
\end{array}\right), \boldsymbol{M}=\left(\begin{array}{c|c}
\mathbf{0} & C_{(1)} \\
\hline C_{(2)} & \mathbf{0}
\end{array}\right),
$$

being $T_{(j)}, C_{(j)}$ tridiagonal and symmetrical matrices of dimension $N \times N$ (see details in "Appendix D.2").

To solve the linear system (41), we propose an iterative linear solver based on a block version of the Jacobi method:

$$
\left\{\begin{array}{l}
\mathcal{P}_{(0)}=\mathbf{0}, \\
\boldsymbol{D P}_{(s)}=\mathcal{R} \mathcal{H} \mathcal{S}-\boldsymbol{M P}_{(s-1)}, s \in 1,2, \ldots,
\end{array}\right.
$$

where at each iteration $s$, a tridiagonal linear system is efficiently solved by using the Thomas algorithm. As stopping criteria for the iterative linear solver (43), we choose

$$
\left\|\mathcal{P}_{(s+1)}-\mathcal{P}_{(s)}\right\|<\epsilon_{t o l} .
$$

In the subsequent numerical test performed in this work, $\epsilon_{t o l}$ is set to $10^{-8}$.

Once the non-hydrostatic pressure terms $\boldsymbol{P}^{(k)}$ have been computed, the velocities $\boldsymbol{U}^{(k)}$ can be updated from Eqs. (36a-36d), wherein a similar way, a second order point value approximation in the centre of the cell will be used.

Remark 7 Although $T_{(j)}$ and $C_{(j)}$ are symmetrical matrices, in general the same does not hold true for the matrix $\boldsymbol{A}$. A particular analysis of the singularity of the matrix $\boldsymbol{A}$ has been made for the case of small water height in "Appendix D.3", but the same question remains open for the general case. Nevertheless, the numerical experiments performed show that the system is indeed invertible. Furthermore, the fast convergence of the iterative method used to solve the linear system let us make the hypothesis that it has a good condition number.

Remark 8 The proposed iterative linear solver has some advantages over other standard linear solvers such as Krylov gradient-based methods (e.g., biconjugate gradient stabilized method) or $L U$ factorization. A short discussion is summarized:

- The proposed linear solver reaches the convergence in less than 20 iterations for the numerical tests showed in this work.

- It can be easily implemented, and it is matrix-free, since only tridiagonal systems have to be solved, which is done with the matrix-free Thomas algorithm. This makes this algorithm extremely convenient for an implementation on GPU architectures, where memory is an important issue. 
- A $L U$ factorization method it is not feasible since the matrix of the linear system $\boldsymbol{A}$ depends on time step. This will demand a considerable computational effort. Moreover, this choice is more memory consuming than the iterative method proposed in this work in (43).

- Let us denote by $\widetilde{\mathcal{P}}$ a convenient reorganization of the variable $\mathcal{P}$, such that

$$
\widetilde{\mathcal{P}}=\left(p_{b, 1}^{(k)}, p_{I, 1}^{(k)}, \ldots p_{b, N}^{(k)}, p_{I, N}^{(k)}\right)^{t} .
$$

Then the corresponding associated matrix $\tilde{\boldsymbol{A}}$ is symmetric and penta-diagonal. This organization is more convenient for an iterative Krylov space based method. Nevertheless, we have found that this methods are more memory and time consuming, even with preconditioners, when compared with the iterative solver proposed in this work.

- Considering future works and the extension of the scheme to bidimensional domains, this will lead to a Poisson-like equation in two dimensions. The proposed algorithm can be combined with a Jacobi or Jacobi relaxation method (see [3]) to take into account this extension.

\subsection{Final Numerical Scheme}

For the sake of clarity, a guideline of the final numerical scheme will be highlighted in what follows. For every $k$ th stage $k \in\{1,2\}$ of the Runge-Kutta method, the problem (18) is split into two parts. A two-step projection-correction method is used:

- Finite volume step (solving the hydrostatic system): From (34), solve explicitly the hyperbolic system at the $k$ th stage of the Runge-Kutta:

$$
\boldsymbol{U}^{(\widetilde{k})}=\boldsymbol{U}^{(k-1)}-\frac{\Delta t}{\Delta x}\left(D_{i+1 / 2}^{(k-1),-}+D_{i-1 / 2}^{(k-1),+}+\mathcal{I}_{i}\right),
$$

by means of a PVM path-conservative finite volume scheme (34) combining a MUSCL reconstruction operator to obtain the intermediate value $\boldsymbol{U}^{(\widetilde{k})}$ in the two-step projectioncorrection method that contains the numerical solution of the underlying hyperbolic system.

- Finite Difference step (non-hydrostatic pressure correction):

- Solve the Poisson-like Eqs. (37) and (38) to obtain the non-hydrostatic pressures

$$
\boldsymbol{P}^{(k)}=\left(\begin{array}{c}
p_{b}^{(k)} \\
p_{I}^{(k)}
\end{array}\right) .
$$

To do so, compact centred second order finite differences are used for the discretization of the derivatives that appear in (37)-(38), and a linear system is solved to obtain $\boldsymbol{P}^{(k)}$.

- With the computed non-hydrostatic pressure terms $\boldsymbol{P}^{(k)}$, the velocities can be updated from (36a)-(36d), where in a similar way, a second order point value approximation in the centre of the cell will be used to compute the non-hydrostatic contribution.

Finally,

$$
\boldsymbol{U}^{n+1}=\frac{1}{2} \boldsymbol{U}^{n}+\frac{1}{2} \boldsymbol{U}^{(2)}
$$




\subsection{Boundary Conditions}

In this work, three types of Boundary Conditions (BC) have been considered: periodic, outflow and generating/absorbing BCs.

- Periodic BCs: given a spatial domain subdivided into a set of $N$ computing cells, cell $I_{1}$ and $I_{N}$, which are the extremes of the domain, are considered as the same cell. This means that its neighbour cells are $I_{N-1}$ on the left and $I_{2}$ on the right.

- Outflow BCs: homogeneous Neumann conditions are applied on the left and right boundaries. Since we use a second order MUSCL scheme, the usage of one ghost cell $I_{0}, I_{N+1}$ at each boundary is required in order to determine the values of the closest nodes to the boundary. The values of the variables at the ghost cells are extrapolated from the adjacent cells

$$
\boldsymbol{U}_{0}=\boldsymbol{U}_{1}, \boldsymbol{P}_{0}=\boldsymbol{P}_{1}, \boldsymbol{U}_{N+1}=\boldsymbol{U}_{N}, \boldsymbol{P}_{N+1}=\boldsymbol{P}_{N} .
$$

Nevertheless, reflections at the boundaries might perturb the numerical solution at the inner domain. As in many other works (see $[43,45,53,60]$ among others), this condition is sometimes supplemented here with an absorbing $\mathrm{BC}$, described below.

- Generating/absorbing BCs: Periodic wave generation as well as absorbing BCs are achieved by using a generation/relaxation zone method similar to the one proposed in [53].

Generation/absorption of waves is achieved by simply defining a relaxation coefficient $0 \leq m(x) \leq 1$, and a target solution $\left(\boldsymbol{U}^{*}, \boldsymbol{P}^{*}\right)$. Given a width $L_{R e l}$ of the relaxation zone on each boundary, we define $k_{R e l}$ as the first natural number that $k_{R e l} \Delta x \geq L_{R e l}$. The solution within the relaxation zone is then redefined to be, $\forall i \in\left\{1, \ldots, k_{R e l}, N-k_{r e l}, \ldots N\right\}$ :

$$
\begin{aligned}
\tilde{\boldsymbol{U}}_{i} & =m_{i} \boldsymbol{U}_{i}+\left(1-m_{i}\right) \boldsymbol{U}_{i}^{*} \\
\tilde{\boldsymbol{P}}_{i} & =m_{i} \boldsymbol{P}_{i}+\left(1-m_{i}\right) \boldsymbol{P}_{i}^{*},
\end{aligned}
$$

where $m_{i}$ is defined as

$$
m_{i}=\sqrt{1-\left(\frac{d_{i}}{L_{R e l}}\right)^{2}},
$$

where $d_{i}$ is the distance between the centre of the cells $I_{i}$ and $I_{1}$ (or $I_{i}$ and $I_{N-k}$ ), in the case of $i \in\{1, \ldots, k\}$ (or $i \in\{N-k, \ldots, N\}$ ).

In our numerical experiments, we set

$$
L \leq L_{\text {Rel }} \leq 1.5 L
$$

being $L$ the typical wavelength of the outgoing wave.

Absorbing BC is the special case $\boldsymbol{U}_{i}^{*}=\boldsymbol{P}_{i}^{*}=0$, that will dump all the waves passing through.

Remark 9 In Sect. 6, some laboratory tests are studied and the effects of the friction of the water with the bottom $H$ must be considered. In order to include friction with the bottom, a Manning empirical formula is added to the right hand side of (18) as a source term

$$
\boldsymbol{S}(\boldsymbol{U})=\left(\begin{array}{lllll}
0 & -\tau & 0 & 0 & 0
\end{array}\right)^{t}, \tau=g l_{1} h n^{2} \frac{u_{1}\left|u_{1}\right|}{\left(l_{1} h\right)^{4 / 3}},
$$


and it is discretized in a semi-implicit way at the end of the second step of the proposed numerical scheme, at each Runge-Kutta stage.

Remark 10 Since non-hydrostatic and friction terms appear only in the momentum equations, the final numerical scheme is well-balanced for the water at rest solution and positive preserving for the water height.

Remark 11 The extension of the proposed numerical scheme to the case of moving bottom is straightforward.

Remark 12 It is well known the fact that higher order schemes should be used when discretizing PDEs of dispersive nature, such as Serre-Green-Naghdi [40], Nwogu [58] among others, since the leading numerical error of the numerical scheme is of dispersive nature (see $[25,45,48,60]$ among many other works where Boussinesq-type systems are discretized). In this case, a linear stability Von-Neumann analysis shows that second order schemes, as well as first order splitting schemes, may destroy the continuum dispersive relation of the original system. Although we did not perform such an analysis in this work, we obtain positive results in the numerical tests. Concretely, Sect. 6.2.2 includes a convergence analysis. Results in Table 1 show that the numerical scheme is formally second-order in space and time. We have also measured the impact on the phase velocity for the propagation of solitary waves for long integration times with the second order scheme. In Sect. 6.2.3 we have measured the variations on amplitude and celerity of a solitary wave of big amplitude. Results in Table 2 show excellent agreement, obtaining a variation of less than $0.1128 \%$.

\section{Breaking Wave Modelling and Wet-Dry Treatment}

\subsection{Breaking Wave Modelling}

As pointed in [61], in shallow water complex events can be observed related to turbulent processes. One of these processes corresponds to the breaking of waves near the coast. As it will be seen in the numerical tests proposed in this work, the model presented here cannot describe this process without an additional term which allows the model to dissipate the required amount of energy on such situations. When breaking processes occur, mostly close to shallow areas, two different approaches are usually employed when dispersive Boussinesqtype models are considered.

Close to the coast where breaking starts, the SWE propagates breaking bores at the correct speed, since $k H$ is small, and dissipation of the breaking wave is also well reproduced. Due to that, the simplest way to deal with breaking waves, when considering dispersive systems, consists in neglecting the dispersive part of the equation. This means to force the nonhydrostatic pressure to be zero where breaking occurs. Due to that, this technique has the advantage that only a breaking criterion is needed to stop and start it. However, the main disadvantage is that the grid-convergence is not ensured when the mesh is refined, and global and costly breaking criteria should be taken into account (see [43]).

The other strategy, that will be adopted in this work, consists in the dissipation of breaking bores with a diffusive term. Again, breaking criteria to switch on/off the dissipation is needed. Usually, an eddy viscosity approach (see [61]) solves the matter, where an empirical parameter is defined, based on a quasi-heuristic strategy to determine when the breaking occurs. The main difficulty that presents this mechanism is that usually the diffusive term must be discretized implicitly due to the high order derivatives from the diffusion. Otherwise, it will 
lead to a severe restriction on the CFL number. As a consequence, an extra linear system has to be solved, losing efficiency. In any case, this challenge is overcome in [33] for the one layer non-hydrostatic system derived in [71], and a natural extension of this procedure for the two-layer case will be described in what follows.

Let us consider the vertical component of the stress-tensor

$$
\tau_{z z}=2 v \partial_{z} w
$$

where $v(x, z, t)$ is a positive function. We use the same process as in Sect. 2 to depth-average the vertical component of the stress-tensor. By taking into account the incompressibility condition and that the vertical velocity has a linear profile within each layer we obtain

$$
\int_{\mathcal{L}_{\alpha}} \partial_{z} \tau_{z z} d z=-\varsigma_{\alpha} h_{\alpha} \partial_{x} u_{\alpha}
$$

where $\varsigma_{\alpha}=\int_{\mathcal{L}_{\alpha}} \partial_{z} v$.

Let us denote $\mathcal{T}\left(h, \partial_{x} h, H, \partial_{x} H, \boldsymbol{P}, \partial_{x} \boldsymbol{P}\right)$ as $\mathcal{T}\left(\boldsymbol{P}, \partial_{x} \boldsymbol{P}\right)$ for the sake of brevity. Adding the proposed integrated viscosity terms, system (18) reads

$$
\left\{\begin{array}{l}
\partial_{t} \boldsymbol{U}+\partial_{x} \boldsymbol{F}(\boldsymbol{U})+\boldsymbol{B}(\boldsymbol{U}) \partial_{x} \boldsymbol{U}=\boldsymbol{G}(\boldsymbol{U}) \partial_{x} H+\mathcal{T}\left(\boldsymbol{P}, \partial_{x} \boldsymbol{P}\right)-\boldsymbol{\tau}+\boldsymbol{R}_{b}\left(\boldsymbol{U}, \partial_{x} \boldsymbol{U}, \varsigma_{1}, \varsigma_{2}\right), \\
\mathcal{B}\left(\boldsymbol{U}, \partial_{x} \boldsymbol{U}, H, \partial_{x} H\right)=\mathbf{0},
\end{array}\right.
$$

where

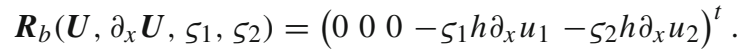

Now we close the system defining $\varsigma_{\alpha}(x, t)$. The linear combination of the non-hydrostatic pressures, $p_{b}$ and $p_{I}$, at the fourth and fifth component of $\mathcal{T}\left(\boldsymbol{P}, \partial_{x} \boldsymbol{P}\right)$, can be expressed in terms of $u_{\alpha}$ and its derivatives $\partial_{x x} u_{\alpha}, \partial_{x t} u_{\alpha}, \partial_{x} u_{\alpha}$ and $\partial_{t} u_{\alpha}$. The proposed election of $\varsigma_{\alpha}$ in this work is based on the idea of cancelling those aforementioned $\partial_{x} u_{\alpha}$ terms with $-\varsigma_{\alpha} h_{\alpha} \partial_{x} u_{\alpha}$. The procedure is detailed in "Appendix C". The following definition, for the case of immobile bed $\partial_{t} H=0$, is proposed:

$$
\left\{\begin{array}{l}
\varsigma_{1}=w_{1}-3 u_{1} \partial_{x} H \\
\varsigma_{2}=w_{2}+3 u_{2} \partial_{x} z_{I}
\end{array}\right.
$$

Note that fourth and fifth components of $\boldsymbol{R}_{b}$ are essentially first order derivatives of $u_{1}, u_{2}$, and can be discretized explicitly.

Finally, a breaking criteria to switch on/off the dissipation is needed. Following a natural and simpler extension of the criteria proposed by [61], wave energy dissipation associated with breaking begins when $\left|\partial_{x}\left(l_{1} h u_{1}+l_{2} h u_{2}\right)\right| \geq U_{1}$ and continues as long as $\mid \partial_{x}\left(l_{1} h u_{1}+\right.$ $\left.l_{2} h u_{2}\right) \mid \geq U_{2}$, where

$$
U_{1}=B_{1} \sqrt{g h}, \quad U_{2}=B_{2} \sqrt{g h},
$$

denote the flow speeds at the onset and termination of the wave-breaking process and $B_{1}, B_{2}$ are calibration coefficients that should be calibrated through laboratory experiments. In this work, as in [61], we use $B_{1}=0.5$ and $B_{2}=0.15$ for al the test cases studied.

Note that the breaking criteria to switch on/off the dissipation is a simplified version of the one proposed in [61], that includes some improvements such as: taking into account a residence time for the activation/deactivation of the criteria to accounting a continuous 
dissipation; or computing in the breaking criteria $\left|\partial_{x}(h u)\right|$ as $\frac{1}{2}\left(\left|\partial_{x}(h u)\right|+\partial_{x}(h u)\right)$ which would automatically become zero on the back of the crest of the wave.

The breaking mechanism proposed in this work can be considered with this improved breaking criteria given in [61], as well as the one proposed in [43], which are more sophisticated and expensive.

Nevertheless, although we have chosen fast and straightforward breaking criterion, the numerical tests in Sect. 6 will show that this technique performs adequately. Moreover, the simple breaking mechanism considered in this work: corrects the classical overshoot that dispersive models present for the run-up of waves (see Fig. 13); ensures the grid convergence even if breaking mechanism is switching on/off during the experiment. Although the simple breaking criteria do not detect hydraulic jumps, we have observed that the proposed system without the breaking dissipation can handle well with hydraulic jumps. In any case, a more sophisticated breaking detector that reveals hydraulic jumps can be considered as well.

Remark 13 In [33], the one layer non-hydrostatic system, which corresponds to the election of $l_{2}=0$ for the presented system in this work, is studied. There, the classical eddy viscosity model approach for breaking waves is considered. The eddy viscosity model, which consists in a diffusion term in the horizontal momentum equation, is reinterpreted as a term of the form $\varsigma h \partial_{x} u$ at the vertical momentum equation. The resulting terms in this work in $\boldsymbol{R}_{b}$ can be seen as the natural extension of the idea presented in [33], and thus as a natural extension of the classic eddy viscosity models for small-scale turbulence.

\subsection{Wet-Dry Treatment}

For the computation of $\boldsymbol{U}^{(\widetilde{k})}$ in the finite volume discretization of the underlying hyperbolic system, a wet-dry treatment adapting the ideas described in [18] is applied. The key to the numerical treatment for wet-dry fronts with emerging bottom topographies relies on:

- The hydrostatic pressure terms $g l_{1} h \partial_{x} \eta, g l_{2} h \partial_{x} \eta$ at the horizontal velocity equations are modified for emerging bottoms to avoid spurious pressure forces (see [18]).

- To compute velocities appearing in Sect. 4.2 from the discharges, very small values for the water height $h$ may appear. To overcome the difficulties due to large round-off errors in computing the velocities $u_{\alpha}, w_{\alpha}$ appearing in the scheme, we define the velocities analogously as in [44] applying the desingularization formula

$$
u_{\alpha}=\frac{\sqrt{2} h h u_{\alpha}}{\sqrt{h^{4}+\max \left(h^{4}, \epsilon^{4}\right)}}, w_{\alpha}=\frac{\sqrt{2} h h w_{\alpha}}{\sqrt{h^{4}+\max \left(h^{4}, \epsilon^{4}\right)}}, \alpha \in\{1,2\},
$$

which gives the exact value of $u_{\alpha}$ and $w_{\alpha}$ for $h \geq \epsilon$, and gives a smooth transition of $u_{\alpha}$ and $w_{\alpha}$ to zero when $h$ tends to zero without truncation. In this work we set $\epsilon=10^{-5}$ for the numerical tests. A more detailed discussion about the desingularization formula can be seen in [44].

In the second step of the projection method, no special treatment is required due to the rewriting of the two incompressibility equations, that have been multiplied by $h^{2}$, in terms of discharges. In presence of wet-dry fonts, non-hydrostatic pressure $\mathcal{P}$ vanishes and no artificial truncation up to a threshold value is needed. In "Appendix D.3", a particular analysis is detailed for the case of

$$
h=\epsilon, u_{\alpha}=w_{\alpha}=0, \partial_{x} H=m .
$$


We also assume that

$$
\epsilon^{2} \approx 0, \epsilon \cdot m \approx 0
$$

to simplify the analysis. A more general case is considered in "Appendix D.3". In this case, the Right hand side of the linear system becomes zero, and the matrix $\boldsymbol{A}$ as well as the right hand side $\mathcal{R} \mathcal{H} \mathcal{S}$ defined in (41) simplifies to

$$
\boldsymbol{A}=4\left(m^{2}+1\right)\left(\begin{array}{c|c}
\boldsymbol{I} & -\boldsymbol{I} \\
\hline \gamma_{2} \boldsymbol{I} & \gamma_{1} \boldsymbol{I}
\end{array}\right), \boldsymbol{\mathcal { R }} \mathcal{H} \mathcal{S}=\left(\begin{array}{l}
\boldsymbol{\mathcal { R H }} \mathcal{S}_{1} \\
\boldsymbol{R H}_{\mathcal{S}_{2}}
\end{array}\right)=\left(\begin{array}{l}
\mathbf{0} \\
\mathbf{0}
\end{array}\right) .
$$

Note that $\boldsymbol{A}$ is invertible, since $\gamma_{1}+\gamma_{2} \neq 0$, and thus the non-hydrostatic pressure $\mathcal{P}$ becomes zero.

Remark 14 The definition of the coefficients of the matrix $\boldsymbol{A}$, are given in terms of $l_{1} h, l_{2} h$ and $\eta$, and the topography $H$ does not appear. Thus, in the case of emerging bottoms, the derivatives of $\partial_{x} \eta$ are treated as the pressure terms $g h_{\alpha} \partial_{x} \eta$ in the horizontal velocity equations, and the numerical scheme can deal with a wide variety of complex situations involving wet-dry fronts and emerging topographies, as we will see in the numerical tests.

\section{Numerical Tests}

In this section we present five numerical tests for the model presented in (45) with the breaking and friction terms introduced previously. The chosen tests have been widely studied in dispersive water waves modelling (see $[23,31-33,43,46,49,50,60])$. Thus, this constitutes a proper way to validate the presented dispersive model and the numerical scheme with analytical solutions and real laboratory tests.

The first three tests aim to validate the mathematical model and the numerical scheme with a comparison to analytical or reference solutions. The other two tests show that the solved equations can simulate complex laboratory experiments, showing comparisons with real data.

Comparisons with the one-layer system results, which corresponds to the choice $l_{2}=0$, are presented for some of the considered tests. These comparisons show that the one-layer system does not give good results in some cases. This means that at least a two-layer model should be used.

The quantities of the parameters concerning the following numerical simulations are expressed in units of measure of the International System of Units.

\subsection{Standing Wave in Closed Basin}

First, we shall study the dispersive properties of the model. To do so, we consider the analytical solution from the Stokes linear theory of a standing wave in a closed basin with length $L$ and flat bottom $H=10$. The initial surface elevation is given by

$$
\eta(x, 0)=A \cos \left(\frac{2 \pi x}{L}\right),
$$



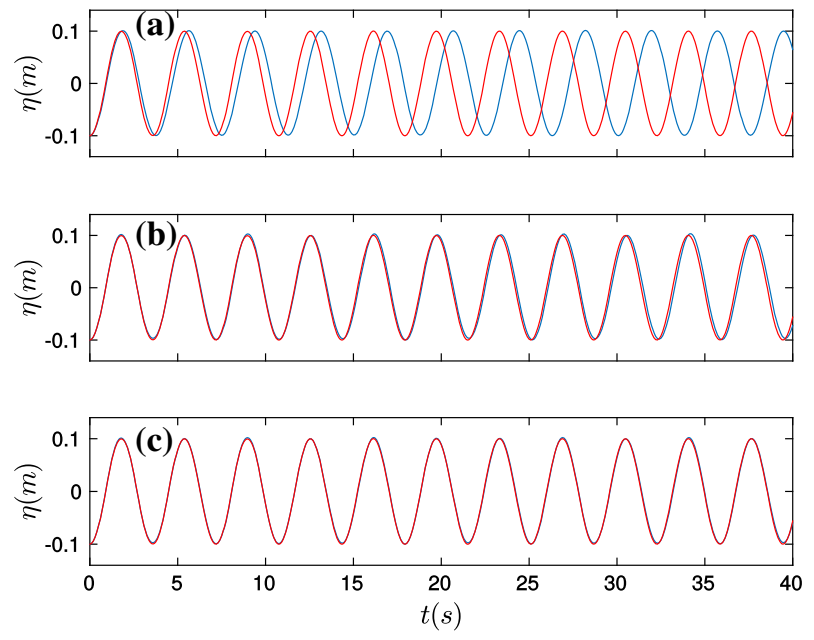

Fig. 4 Comparison of analytical (red) and numerical (blue) for the case $L=20$. In a the numerical results for the one-layer $\left(l_{2}=0\right)$; $\mathbf{b}$ the numerical results with the non-optimized parameter $\left(l_{1}^{(2 L)}, \gamma_{1}^{(2 L)}, \gamma_{2}^{(2 L)}\right)$; $\mathbf{c}$ the numerical results with the optimized parameters $\left(l_{1}^{(5)}, \gamma_{1}^{(5)}, \gamma_{2}^{(5)}\right)$ (Color figure online)

where $A=0.1$ is the amplitude of the standing wave. The wavelength is the same than the length of the basin. This test case has been studied by various authors. For instance, Casulli and Stelling [23] use more than 20 vertical layers in order to correctly simulate wave dispersion. Using Keller-box scheme, Stelling and Zijlema [64] obtained good agreement with the analytical solution by using two layers. Using a $\sigma$-coordinates discretization of the free-surface Euler equations, [50] shows excellent agreement with three layers.

To check the performance of the two proposed improved parameters, we will show two cases: $L=20 / n, n=1,3$. Since $k H=20 \pi / L=n \pi$, the waves are highly dispersive. From the dispersion relationship, we know that $2 \pi / T=\sqrt{g k \tanh (k H)}$, and we can calculate the wave period $T$. The Stokes first order solution for this standing wave is

$$
\eta(x, t)=\frac{A}{2} \cos (k x) \cos \left(\frac{2 \pi}{T} t\right) .
$$

As in [51], a grid $\Delta x=0.2 / n$ that corresponds to 100 cells per wavelength for both cases of $n=1,3$. A $C F L=0.45$ is employed. Periodic boundary conditions are imposed. The gravity acceleration is set to $g=9.81$ and the simulation time is $40 \mathrm{~s}$. The test is performed with the one-layer system that corresponds to $l_{2}=0$, and with the two-layer system proposed in this work for some values of the optimization parameters that will be detailed.

Figure 4 shows the comparison of the time series between the analytical solution for the case of $L=20$ and the numerical solution, at $x=L / 2$. In this case, $k H=\pi$, and the two-layer system with the non-optimized parameters $\left(l_{1}^{(2 L)}, \gamma_{1}^{(2 L)}, \gamma_{2}^{(2 L)}\right)$ gives good results. Nevertheless, the optimized parameters $\left(l_{1}^{(5)}, \gamma_{1}^{(5)}, \gamma_{2}^{(5)}\right)$ improve the results and the solution matches perfectly with the analytical reference solution. This case highlights that the onelayer system cannot reproduce well these dispersive waves. This is due to the poor accuracy of the linear dispersion relation that the one-layer has for $k H \approx \pi$.

The case $L=20 / 3$ gives $k H \approx 9.42$ and becomes more challenging. Figure 5 shows comparisons of the time series at $x=L / 2$. In this case, the two-layer model with the non- 

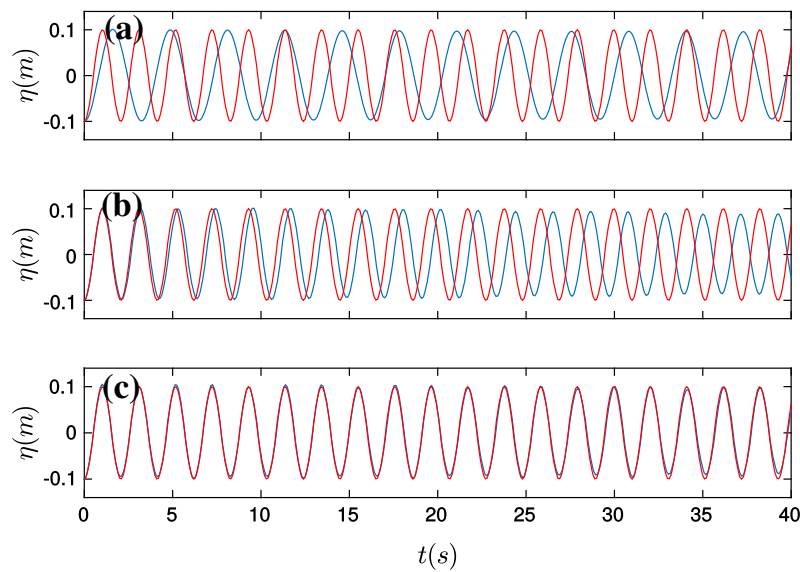

Fig. 5 Comparison of analytical (red) and numerical (blue) for the case $L=20 / 3$. In a the numerical results for the one-layer $\left(l_{2}=0\right)$; $\mathbf{b}$ the numerical results with the non-optimized parameters $\left(l_{1}^{(2 L)}, \gamma_{1}^{(2 L)}, \gamma_{2}^{(2 L)}\right)$; $\mathbf{c}$ the numerical results with the optimized parameters $\left(l_{1}^{(15)}, \gamma_{1}^{(15)}, \gamma_{2}^{(15)}\right)$ (Color figure online)

optimized parameters cannot maintain the correct frequency, and it quickly loses the initial trim, which is also missed from the beginning in the case of the one-layer system. Figure 5 also shows the results with the presenting improved parameters $\left(l_{1}^{(15)}, \gamma_{1}^{(15)}, \gamma_{2}^{(15)}\right)$ with an excellent agreement. The numerical test shows the ability of the optimized model presented here to deal with a wide range of waves.

\subsection{Eulerian Solitary Gravity Waves}

This subsection is split into three parts: first, we prove that with the proposed set of parameters, $\left(l_{1}^{(5)}, \gamma_{1}^{(5)}, \gamma_{2}^{(5)}\right)$, the model can propagate eulerian solitary gravity waves of amplitude up to $A=0.6$ in a more accurate way than the original two-layer system, which corresponds to the election of the parameters $\left(l_{1}^{(2 L)}, \gamma_{1}^{(2 L)}, \gamma_{2}^{(2 L)}\right)$. Second, the numerical accuracy of the scheme presented in the paper is verified by performing a grid convergence study on the solitary wave described. Third, a solitary wave is propagated in a very long integration of time for different grid sizes, showing the excellent performance of the numerical scheme in such situations. In the subsequent test, we consider periodic boundary conditions and the minmod limiter for the SWE step of the numerical scheme is turned off to measure the order of accuracy of the numerical scheme.

\subsubsection{Eulerian Solitary Gravity Waves: Description and Choosing the Parameters}

In this test, we study the case of solitary waves with very large amplitudes. Solitary waves travel at constant speed with permanent shape, which can exist due to the balance between non-linear and linear wave dispersion.

When the amplitude of a solitary wave is small compared to the typical depth $H$, the characteristic length of the wave is relatively large. Thus, the solitary wave is a long wave and weakly-nonlinear weakly-dispersive models such as as [16], or [71] can simulate the wave correctly. However, as the amplitude increases, the wavelength decreases, and therefore 
higher-order non-linear and dispersive effects become more important. This phenomenon will be investigated here with the two-layer model.

To compute the initial condition in the form of a solitary wave for the model, we use the numerical method presented in [26,30]. The tool given in [26] provides the free-surface $\eta_{E}$, and profiles at a given level $z=s h-H$ for $s$ from 0 to 1 , for horizontal and vertical velocities $u_{E}(z), w_{E}(z)$. Note that due to the form of the presented numerical scheme, no initial condition for the non-hydrostatic pressure is needed. Thus, given an election of the free-parameter $l_{1}$, we compute the initial condition for the depth-integrated horizontal and vertical velocities within each layer. To do that, we use Gaussian quadrature points of third order.

For the two solitary waves examined in this section, with amplitudes of $A_{1}=0.2, A_{2}=$ 0.6 , a grid length $\Delta x=0.01$ and a CFL number 0.4 is employed. The bathymetry is constant $H=1.0$, the gravity acceleration is set to $g=1$, and the simulations are carried out in a channel of $500 \mathrm{~m}$ during $350 \mathrm{~s}$. The tests are performed with the one-layer system, with the two-layer with the improved parameters $\left(l_{1}^{(5)}, \gamma_{1}^{(5)}, \gamma_{2}^{(5)}\right)$ and with the non-improved parameters $\left(l_{1}^{(2 L)}, \gamma_{1}^{(2 L)}, \gamma_{2}^{(2 L)}\right)$. Only the results obtained with the parameters $\left(l_{1}^{(5)}, \gamma_{1}^{(5)}, \gamma_{2}^{(5)}\right)$ will be commented.

As this wave solution is not an analytical solitary-wave solution of the two-layer model when the numerical simulation is started the solitary wave drops some waves at the tail. Due to this initial fluctuation in waveform, the wave decreases in amplitude and so the celerity. Nevertheless, the solitary wave, which moves rapidly due to its large amplitude, eventually leaves this tail far behind and reaches a steady form. This initial mismatch effects also occur when considering approximated solitary wave solutions for Boussinesq systems (see [46]).

Figure 6 shows the evolution of the amplitude and speed of both solitary waves. The steady amplitudes and speeds reached for both cases are $\tilde{A}_{1}=0.197, \tilde{c}_{A_{1}}=1.094$ and $\tilde{A}_{2}=0.588, \tilde{c}_{A_{2}}=1.243$. Steady states are achieved not so far. This means that waves travel at a constant speed even in the case of large amplitudes, which is one of the abilities of the two-layer model that is intended to show in this test.

It can be seen that celerities of the simulations $\tilde{c}_{A_{1}}$ and $\tilde{c}_{A_{2}}$ are both in agreement with the reference celerities that can be seen in [30].

Figure 7 shows the numerical solutions obtained with the two-layer model at $350 \mathrm{~s}$ shifted by $\left(x-350 c_{A_{i}}\right)$ meters and compared with the corresponding initial conditions. For all the cases, the two-layer model matches the initial conditions to a very high accuracy. It can be observed that a better fit is obtained when considering the two-layer model with the improved parameters.

Figure 8 shows the results obtained with the one-layer system corresponding to $l_{2}=0$ for the case of $A_{2}=0.6$. In this case, although a steady amplitude and celerity are also reached, it can be observed that the system can not maintain the initial amplitude. Thus the celerity decreases and the computed solitary wave is delayed in approximately $5 \mathrm{~m}$ at $350 \mathrm{~s}$.

It can be stated from these comparisons that the two-layer model presented here captures, to a highly accurate degree, the physics of a non-linear solitary wave.

\subsubsection{Convergence Analysis}

Hereinafter, the set of parameters $\left(l_{1}^{(5)}, \gamma_{1}^{(5)}, \gamma_{2}^{(5)}\right)$ are used to verify that the proposed numerical scheme is second order accurate in both space and time. Some numerical simulations for different grids have been computed up to time $t=30 \mathrm{~s}$ for the case of the solitary wave of amplitude $A=0.2$. 

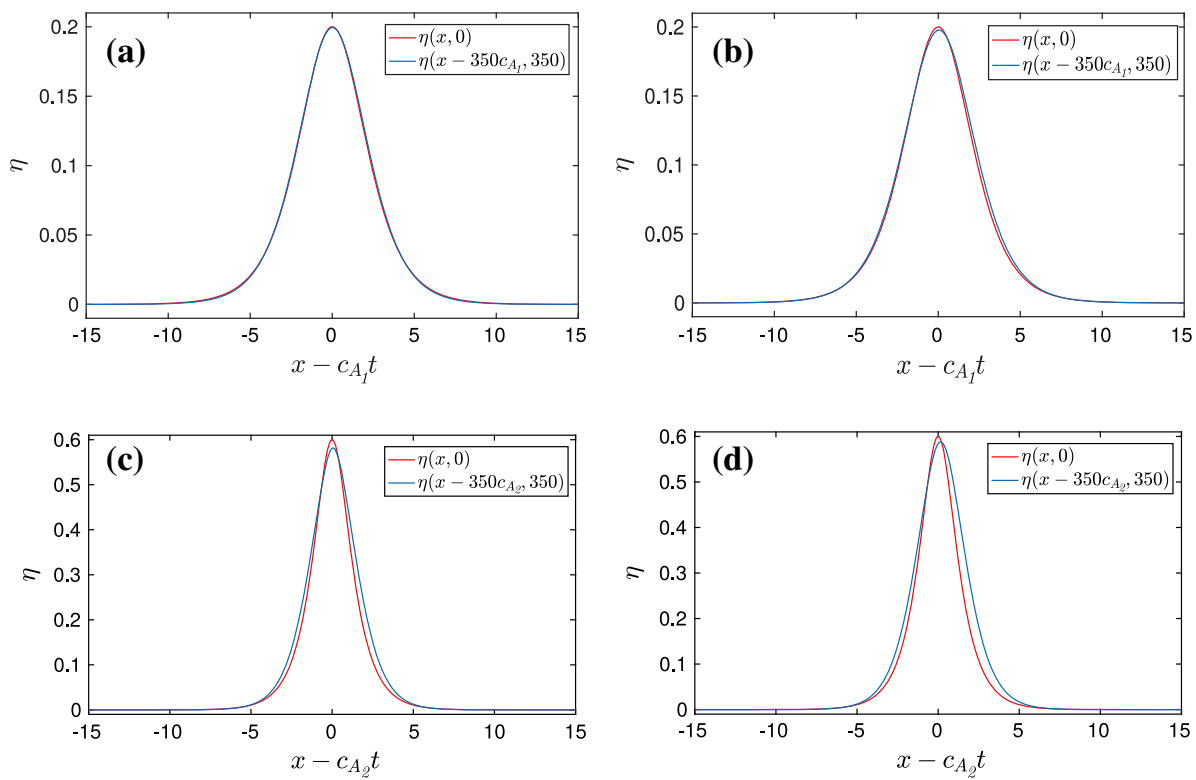

Fig. 6 Comparison of surface at time $t=350$ shifted by $\left(x-c_{A_{i}} t\right) m$ (blue) and initial condition (red). a, c The comparison with the improved parameters $\left(l_{1}^{(5)}, \gamma_{1}^{(5)}, \gamma_{2}^{(5)}\right)$ for the trial $A_{1}=0.2$ and $A_{2}=0.6$ respectively. b, $\mathbf{d}$ The comparison with the non-improved parameters $\left(l_{1}^{(2 L)}, \gamma_{1}^{(2 L)}, \gamma_{2}^{(2 L)}\right)$ for the trial $A_{1}=0.2$ and $A_{2}=0.6$ respectively (Color figure online)
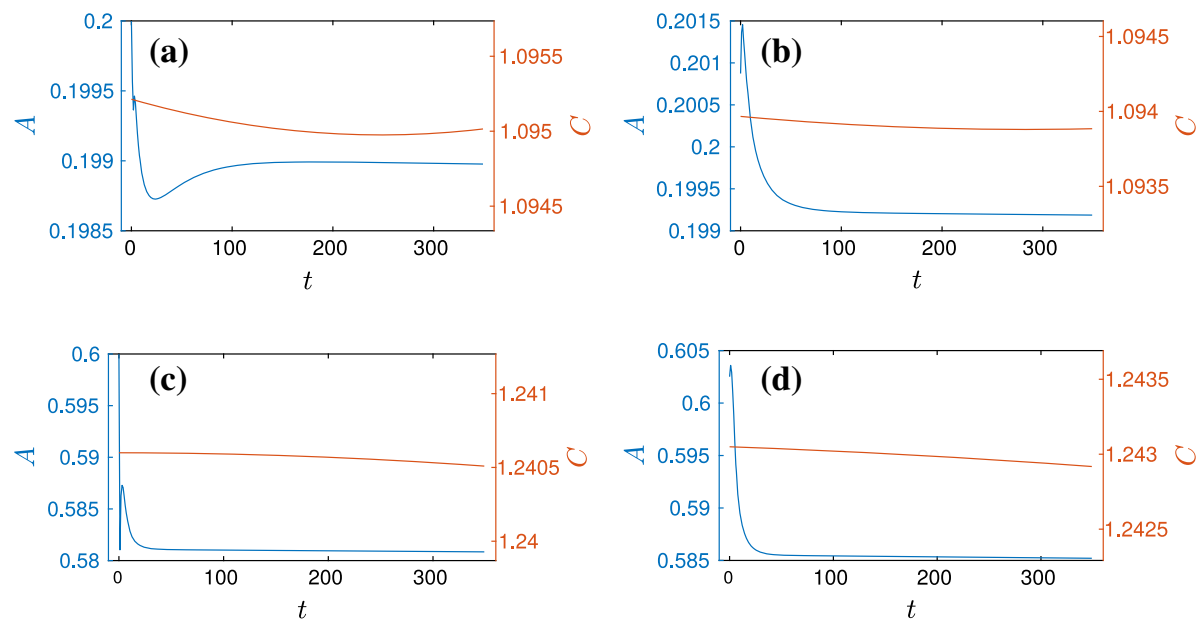

Fig. 7 Variation through the time of the amplitude (blue) and celerity (red). a, c The comparison with the improved parameters $\left(l_{1}^{(5)}, \gamma_{1}^{(5)}, \gamma_{2}^{(5)}\right)$ for the trial $A_{1}=0.2$ and $A_{2}=0.6$ respectively. $\mathbf{b}, \mathbf{d}$ The comparison with the non-improved parameters $\left(l_{1}^{(2 L)}, \gamma_{1}^{(2 L)}, \gamma_{2}^{(2 L)}\right)$ for the trial $A_{1}=0.2$ and $A_{2}=0.6$ respectively (Color figure online) 

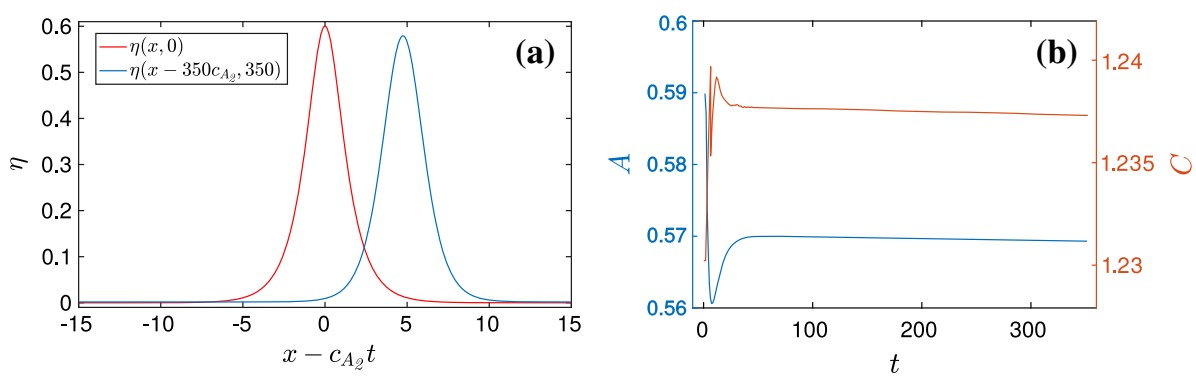

Fig. 8 a Comparison of surface at time $t=350$ shifted by $\left(x-c_{A_{2}} t\right) m$ (blue) and initial condition (red) for the trial $A=0.6$ with the one-layer system. b The variation through the time of the amplitude (blue) and celerity (red) for the trial $A_{2}=0.6$ with the one-layer system (Color figure online)

Table $1 L^{1}$ errors and convergence rates for the solitary wave problem of amplitude $A=0.2$

\begin{tabular}{lllll}
\hline$\Delta x$ & $L^{1}$ error $h$ & $L^{1}$ order $h$ & $L^{1}$ error $h \boldsymbol{u}$ & $L^{1}$ order $h \boldsymbol{u}$ \\
\hline 0.2 & $3.96 \mathrm{E}-03$ & - & $3.97 \mathrm{E}-03$ & - \\
0.1 & $9.81 \mathrm{E}-04$ & 2.01 & $8.79 \mathrm{E}-04$ & 2.18 \\
0.05 & $2.10 \mathrm{E}-04$ & 2.22 & $2.14 \mathrm{E}-04$ & 2.04 \\
0.025 & $5.14 \mathrm{E}-05$ & 2.03 & $4.38 \mathrm{E}-05$ & 2.29 \\
0.0125 & $1.28 \mathrm{E}-05$ & 2.00 & $1.16 \mathrm{E}-05$ & 1.92
\end{tabular}

The errors have been computed for the variables $h$ and $h \boldsymbol{u}=l_{1} q_{u, 1}+$ $l_{2} q_{u, 2}$

Since the considered solitary wave is not an exact solution for the system, we take as reference solution a numerical simulation at time $t=30 \mathrm{~s}$ for a very fine grid with $\Delta x=$ $3.125 \cdot 10^{-3}$.

Table 1 shows the $L^{1}$ errors and numerical orders of accuracy obtained. We would like to remark that the proposed convergence analysis test is usually done for solitary waves of amplitude less than $A=0.2$ and for an integration time less than $t=30 \mathrm{~s}$ (see $[6,14,60]$ ). Thus, this constitutes a standard test to check the order of accuracy of the numerical scheme for dispersive water waves models.

\subsubsection{A Solitary Wave Over a Long Integration Time: A Grid Analysis}

A solitary wave of amplitude $A=0.6$ is propagated in a very long integration time of $1000 \mathrm{~s}$ for different grid sizes.

Attending to the results in Fig. 9, since the shape of the computed solitary waves are well preserved, we will focus on the delay that the computed numerical waves present with respect to the reference as well as the differences in amplitude. To do that, we will define $e_{A}$ and $e_{S}$ as the relative errors

$$
e_{A}=\frac{\left|A_{\Delta x}-A_{1}\right|}{A_{1}}, e_{S}=\frac{\left|S_{\Delta x}-S_{1}\right|}{S_{1}}
$$

being $A_{1}$ and $A_{\Delta x}$ the maximum amplitude of the reference and computed solitary waves respectively. $S_{1}$ and $S_{\Delta x}$ are the distance covered during $1000 s$ by the reference and computed solitary waves respectively. Table 2 summarizes the results in percent.

The results in Fig. 9 and Table 1 show that with relatively coarse meshes, the numerical scheme can handle with solitary typical waves with a very reasonable delay and decay on 
Table $2 L^{1}$ errors and convergence rates for the Solitary wave problem of amplitude $A=0.2$

\begin{tabular}{lll}
\hline$\Delta x$ & $100 e_{A}(\%)$ & $100 e_{S}(\%)$ \\
\hline 0.2 & 0.1128 & 0.0279 \\
0.1 & 0.1037 & 0.0098 \\
0.05 & 0.0334 & 0.0029 \\
0.025 & 0.0098 & 0.0009 \\
0.0125 & 0.0025 & 0.0002
\end{tabular}

The errors have been computed for the variables $h$ and $h \boldsymbol{u}=l_{1} q_{u, 1}+$ $l_{2} q_{u, 2}$

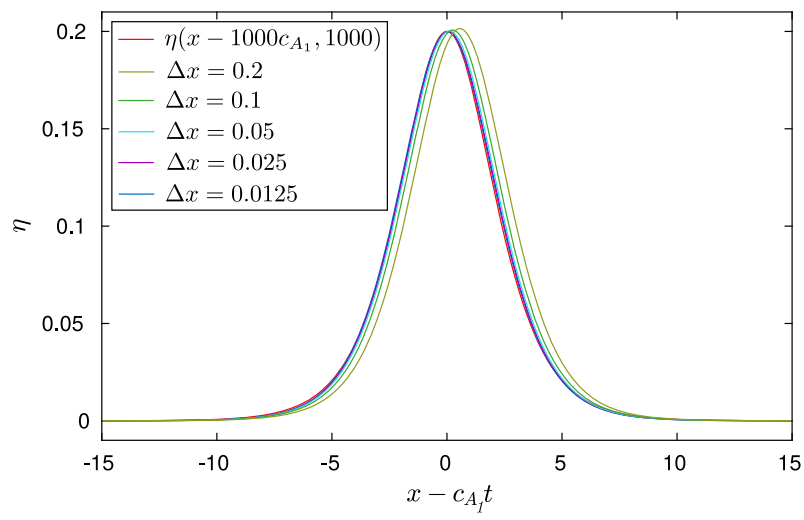

Fig. 9 Comparison of surface at time $t=1000$ shifted by $\left(x-c_{A_{1}} t\right) m$ for different mesh grids and initial condition (in red) for the trial $A=0.2$ (Color figure online)

amplitude less than $0.1128 \%$. Thus, this shows that the numerical scheme, although second order accurate both space and time, does not introduce big errors when propagating waves over long integration times.

\subsection{Head-On Collision of Two Solitary Waves}

The head-on collision of two equal solitary waves is again a standard test for the Boussinesqtype models (see [60,61]). The collision of two solitary waves is equivalent to the reflection of one solitary wave by a vertical wall when the viscosity is neglected.

After the interaction of the two waves, one should ideally recover the initial profiles. The collision of the two waves presents additional challenges to the model due to the sudden change of the non-linear and frequency dispersion characteristics.

We present here the interaction of two solitary waves propagating on a depth of $H=1 \mathrm{~m}$ with amplitude $A=0.4 \mathrm{~m}$. The computational domain is [0,200] and $\Delta x=0.1$ The CFL is set to 0.4 and $g=1$.

The initial condition consists of two solitary waves that travel in opposite directions, placed at $x=50$ and $x=150$ following the same procedure as in Sect. 6.2 for the initial condition. Finally, free-outflow boundary conditions are considered.

Figure 10 shows the collision of the two solitary waves at the midpoint of the domain at time $T=50 / \sqrt{g(A+H)}$, and after the collision both maintain the initial amplitude and 

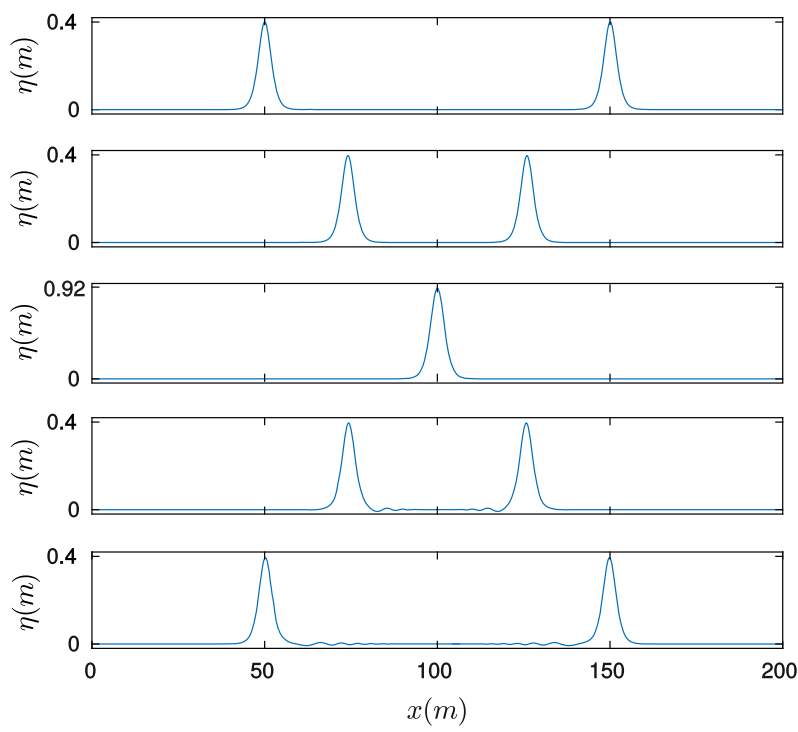

Fig. 10 Head-on collision of two solitary waves at times $T \sqrt{g(A+H)}=0,25,50,75,100$

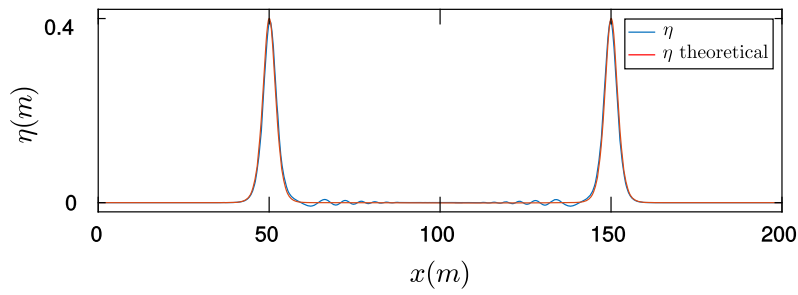

Fig. 11 Head-on collision of two solitary waves. Final state after the interaction. Comparison of the computed free-surface with the exact soliton profiles

the same speed but in opposite directions. Figure 11 shows the final state after the collision compared with the exact profile, showing an excellent agreement.

\subsection{Solitary Wave Run-Up on a Plane Beach}

Synolakis [65] carried out laboratory experiments for incident solitary waves, to study propagation, breaking and run-up over a planar beach with a slope 1:19.85. Many researchers have used this data to validate numerical models. With this test case, we assess the ability of the model to describe shoreline motions and wave breaking when it occurs. Experimental data are available in [65] for surface elevation at different times. The bathymetry of the problem is described in Fig. 12.

A solitary wave of amplitude $A=0.3$ is placed at point $x=20$ as initial condition for the free-surface elevation following the same procedure as in Sect. (6.2). A Manning coefficient of $n_{m}=0.01$ is used in order to define the glass surface roughness used in the experiments. The computational domain is $[-10,40]$ and the numerical parameters used are $\Delta x=0.02$, $C F L=0.9$ and $g=9.81$. Free-outflow boundary conditions are considered. Simulations are carried out with the improved parameters (27). 


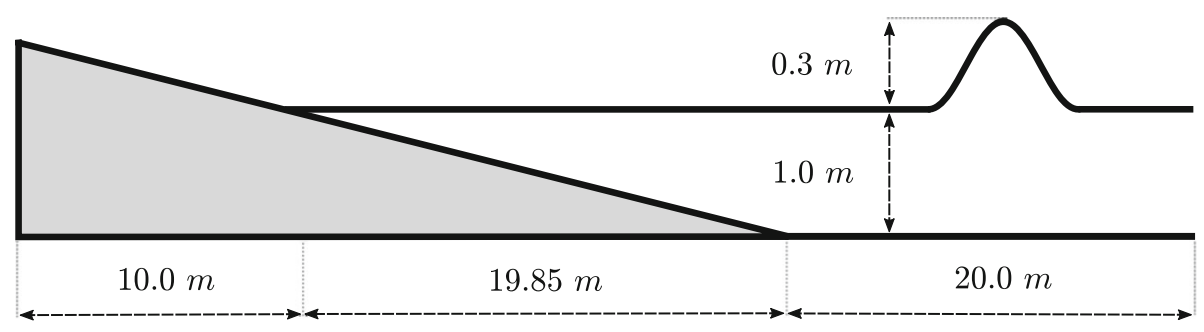

Fig. 12 Sketch of the bathymetry
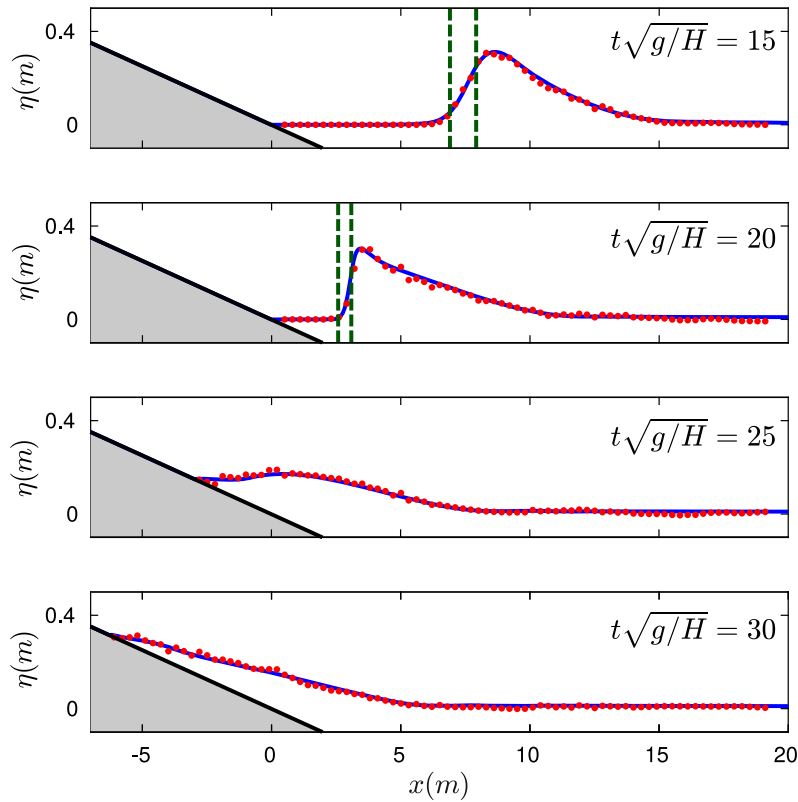

Fig. 13 Comparison of experiments data (red) and simulated ones with breaking terms proposed in (46) (blue) at different times during the run-up. Between bars, regions where breaking mechanism is active are shown (Color figure online)

Figures 13 and 14 show snapshots, at different times, $t \sqrt{g / H}=t_{0}$ where $H=1$, comparing experimental and simulated data. Here we use the proposed $\varsigma_{\alpha}$ for the breaking mechanism described in Sect. 5. Breaking parameters are set to $B_{1}=0.5$ and $B_{2}=0.15$. Fig. 13 also shows where the breaking mechanism is active (region between the bars), and demonstrates the efficacy of the criteria. As we discussed in Sect. 5, the breaking criteria does not take into account the presence of hydraulic jumps. However, Fig. 14 shows that the system without the extra breaking dissipation proposed in this work can handle this situation correctly.

The breaking mechanism also works properly in terms of grid convergence. Figure 15 shows the snapshots at times $t \sqrt{g / H}=15$ (run-up) and $t \sqrt{g / H}=55$ (run-down) for different mesh sizes.

Besides, good results are obtained at maximum run-up, where friction terms play an important role. Note that no additional wet-dry treatment on the second step of the scheme is necessary. 

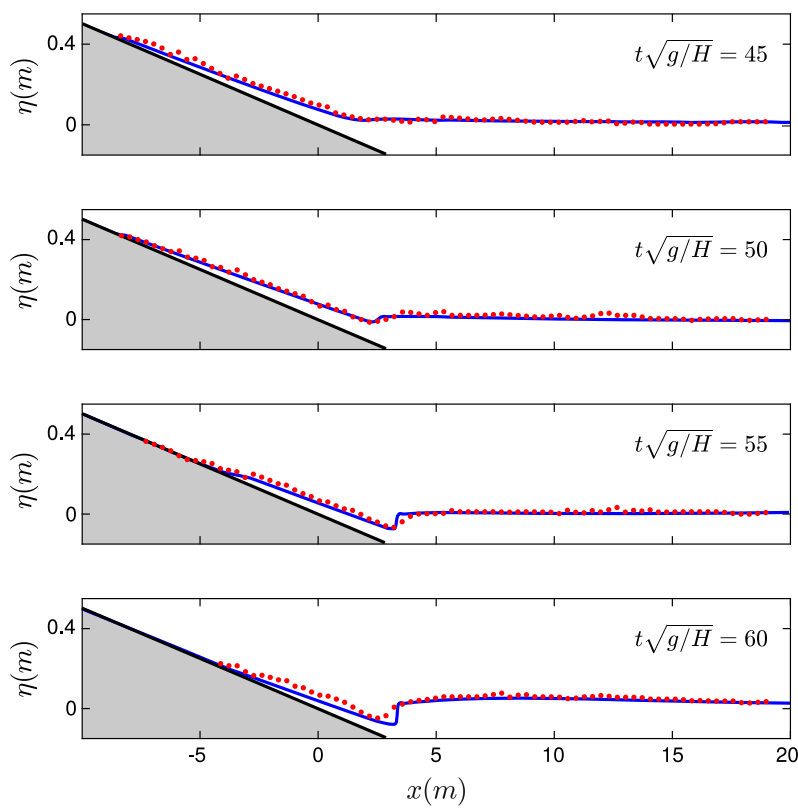

Fig. 14 Comparison of experiments data (red) and simulated ones with breaking terms proposed in (46) (blue) at different times during the run-down (Color figure online)
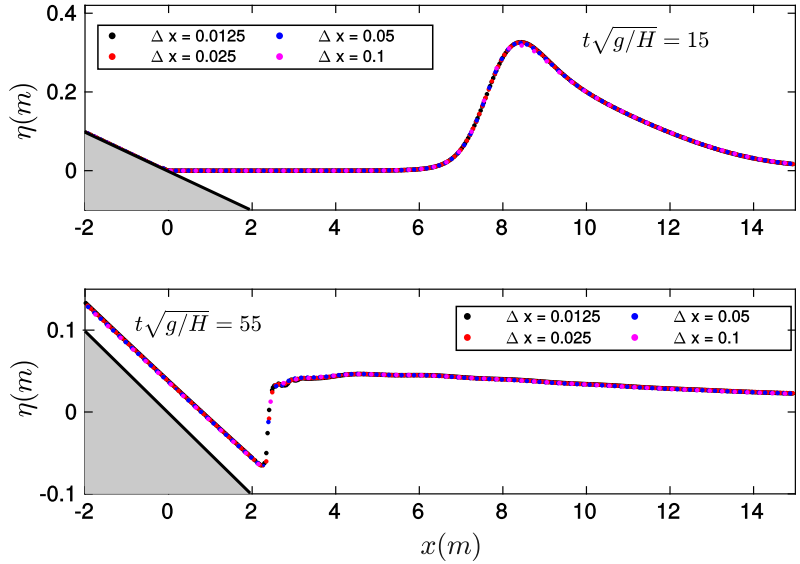

Fig. 15 Comparison of free-surface simulation at times $t \sqrt{g / H}=15$ and $t \sqrt{g / H}=55$ for different mesh sizes

Since the propagated wave in this test is weakly-nonlinear weakly-dispersive, the results obtained with the two-layer system are quite similar to the results obtained with the one-layer system (see [33]). Nevertheless, this test shows that the breaking mechanism introduced in this work, as well as friction terms, perform adequately for the two-layer system. Moreover, the corresponding discretization is robust and can deal with the presence of wet-dry fronts correctly. 


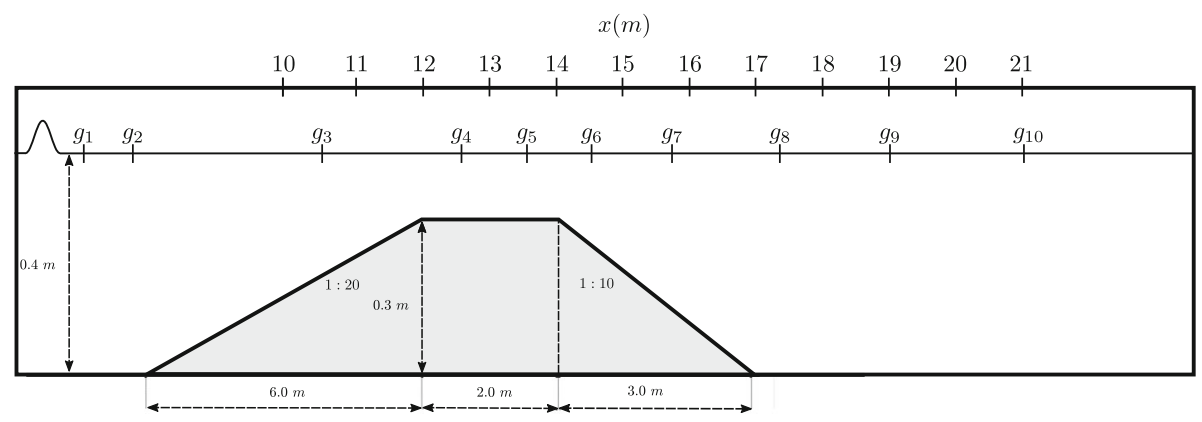

Fig. 16 Periodic waves breaking over a submerged bar. Sketch of the bathymetry

\subsection{Regular Wave Propagation Over a Submerged Bar}

The experiment of plunging breaking periodic waves over a submerged bar by Dingemans [28] is considered here. The experiment studies the frequency dispersion characteristics and nonlinear interaction. As waves propagate over a submerged bar, multiple phenomena occur, such as non-linear shoaling, amplification of higher harmonics and wave breaking.

The one-dimensional domain $[0,30]$ is discretized with $\Delta x=0.01$ and the bathymetry is defined in the Fig. 16. The CFL is set to 0.5 and $g=9.81$. Horizontal and vertical velocities and $\eta$ are set initially to 0 . Boundary conditions correspond to free-outflow at $x=30$ and a sinusoidal wave train for $\eta$ generated at $x=0$. This is done using the target initial condition at the relaxation zone:

$$
\eta^{*}(t)=A \sin \left(\frac{2 \pi}{T} t\right), u_{\alpha}^{*}(t)=\sqrt{g H_{0}} \eta^{*}(t), w_{\alpha}^{*}(t)=p_{b}^{*}(t)=p_{I}^{*}(t)=0, \alpha=1,2
$$

where $A$ and $T$ denotes amplitude and period and $H_{0}=0.4$. We focus on test cases $(a),(b)$ and $(c)$ described in the table below

\begin{tabular}{llll}
\hline Case & $\mathrm{A}(\mathrm{m})$ & $\mathrm{T}(\mathrm{s})$ & \\
\hline (a) & 0.01 & 2.02 & Non-breaking waves \\
(b) & 0.0145 & 2.525 & Breaking waves \\
(c) & 0.0205 & 1.01 & Non-breaking waves \\
\hline
\end{tabular}

Finally, the relaxation length for the initial generating/absorbing boundary conditions are set to $L_{R e l}=2$ for cases $(a)-(c)$.

Depth gauges, which measure the free surface elevation, are placed for cases along $x=$ $g_{i}, g_{1}=4, g_{2}=5.7, g_{3}=10.5, g_{4}=12.5, g_{5}=13.5, g_{6}=14.5, g_{7}=15.7, g_{8}=$ 17.3, $g_{9}=19, g_{10}=21$.

Test $(a)$ and $(c)$ produce, up to the front slope, waves with $k H \approx 0.63$ and $k H \approx 1.58$ respectively. Figure 17 shows the time series of free surface for case $(a)$. The numerical model maintains a good agreement with the experimental data at each station. Minor discrepancies can be observed behind the bar at stations $g_{5}-g_{10}$, where higher harmonics are released. The test case $(a)$ coincides with one of the proposed in [12], and similar good agreement with other numerical models can be found in the literature. Let us remark that in [50] $\sigma$-coordinate 

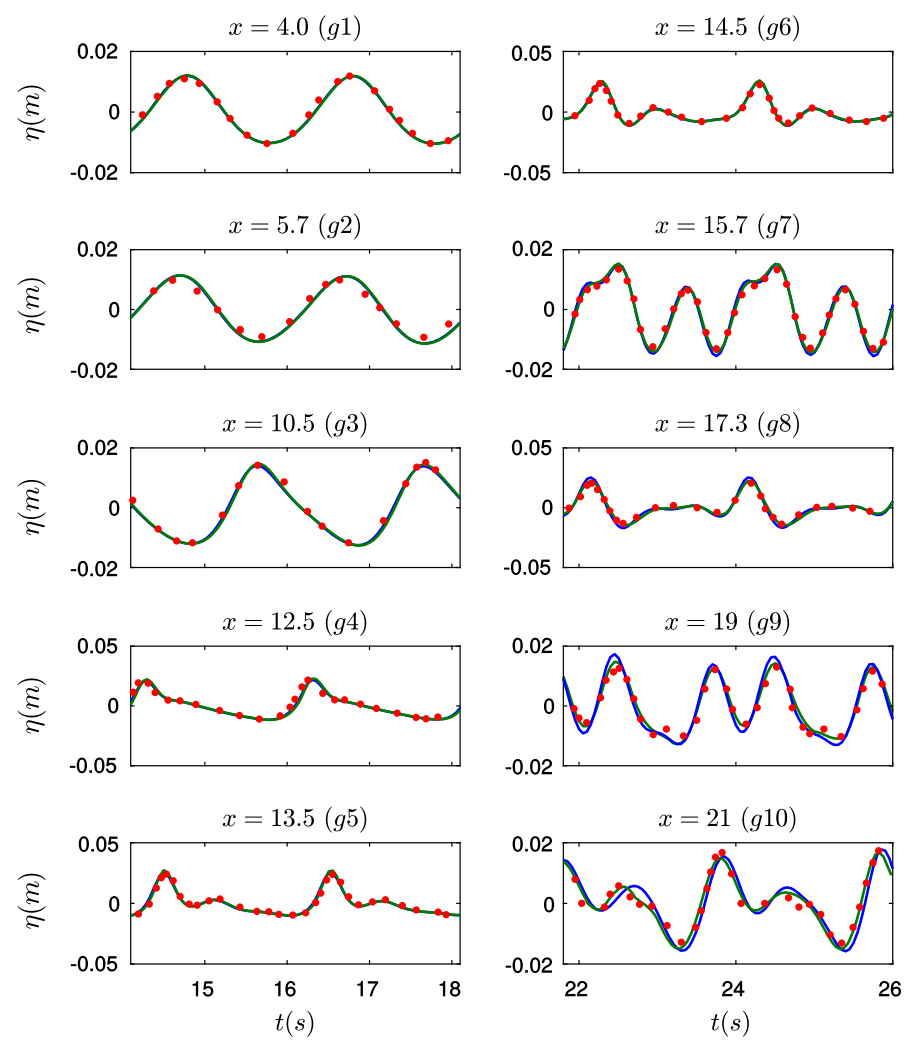

Fig. 17 Comparison of experiment data $(a)$ (red points) and simulated ones with improved dispersive parameters $\left(l_{1}^{(5)}, \gamma_{1}^{(5)}, \gamma_{2}^{(5)}\right)($ green $)$ and $\left(l_{1}^{(2 L)}, \gamma_{1}^{(2 L)}, \gamma_{2}^{(2 L)}\right)$ (blue) (Color figure online)

discretization of the free-surface Euler equations is used. While in that paper the authors need three layers for the test, we can achieve good results with the technique proposed here that only considers two layers. The results in [25] with a three-parameter Green-Naghdi model optimized for uneven bottoms, are also in good agreement. Comparing to that, let us remark that the Green-Naghdi system described there includes high order terms using third order derivatives. We would like to stress the ability of the proposed two-layer system to deal with a wide range of dispersive waves, which is achieved without including high order terms in the system of equations. Only first order derivatives are used here. In [9] the test is also studied with a depth-integrated free-surface two-layer non-hydrostatic system.

The results from case $(c)$ are given in Fig. 18. In this situation, larger values of $k H$ give short waves and make this case more challenging. Due to this fact, waves propagate and typical cnoidal profile can be observed over the top of the bar. This is well reproduced at gauges $g_{1}, g_{2}, g_{3}, g_{4}$. Behind the bar, at $g_{5}, g_{6}, g_{7}$, higher harmonics appear. As we see, a good fit for the amplitude is observed. This test case was studied in [46], where excellent results are obtained at $g_{5}, g_{6}, g_{8}, g_{10}$, with a two-layer system that it has been also optimized up to the second-order nonlinear dispersive analysis.

Figures 17 and 18 also show comparison with $\left(l_{1}^{(5)}, \gamma_{1}^{(5)}, \gamma_{2}^{(5)}\right)$ and $\left(l_{1}^{(2 L)}, \gamma_{1}^{(2 L)}, \gamma_{2}^{(2 L)}\right)$ parameters. One can see a better matching in favour of the former. 

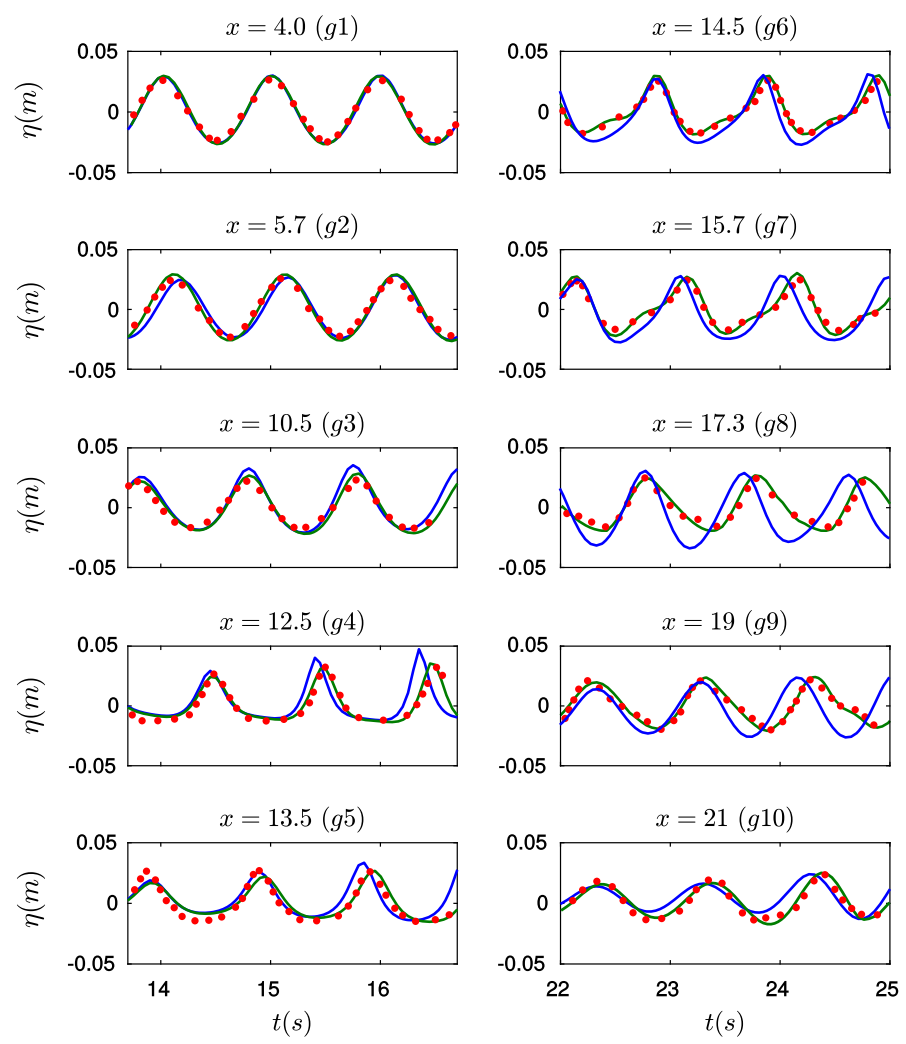

Fig. 18 Comparison of experiment data $(c)$ (red points) and simulated ones with improved dispersive parameters $\left(l_{1}^{(5)}, \gamma_{1}^{(5)}, \gamma_{2}^{(5)}\right)$ (green) and $\left(l_{1}^{(2 L)}, \gamma_{1}^{(2 L)}, \gamma_{2}^{(2 L)}\right)$ (blue) (Color figure online)

The next case presented is $(b)$, where waves start to break on the top of the bar. Numerical time series of surface elevation are shown in Fig. 19 for $\left(l_{1}^{(5)}, \gamma_{1}^{(5)}, \gamma_{2}^{(5)}\right)$. Here we use the proposed $\varsigma_{\alpha}$ for the breaking mechanism described in Sect. 5. Breaking parameters are set to $B_{1}=0.5$ and $B_{2}=0.15$. To evince that a breaking mechanism is needed, the corresponding numerical simulation without the breaking mechanism is also shown in Fig. 19. The test shows the need to consider a breaking mechanism. Before reaching the bar, both simulations coincide as expected, since the wave breaking starts around $x=12$, revealing that the start/stopping criteria performs properly. In all gauges that follow, the wave shape, frequency and amplitude are well reproduced in favour of the simulations with the breaking mechanism. In [29], the test case $(b)$ is performed with a Serre Green-Naghdi system and a different and well-validated breaking mechanism. The results obtained in this work, are in accordance with the ones presented in [29].

The comparison with experimental data emphasizes the need to consider a dispersive model to faithfully capture the shape of the waves near the continental shelf, where a wide range of dispersive waves can be released. Thus, the two-layer system presented in this work can simulate an extensive range of frequencies of dispersive waves, which typically arises in nature from intermediate to shallow waters. 

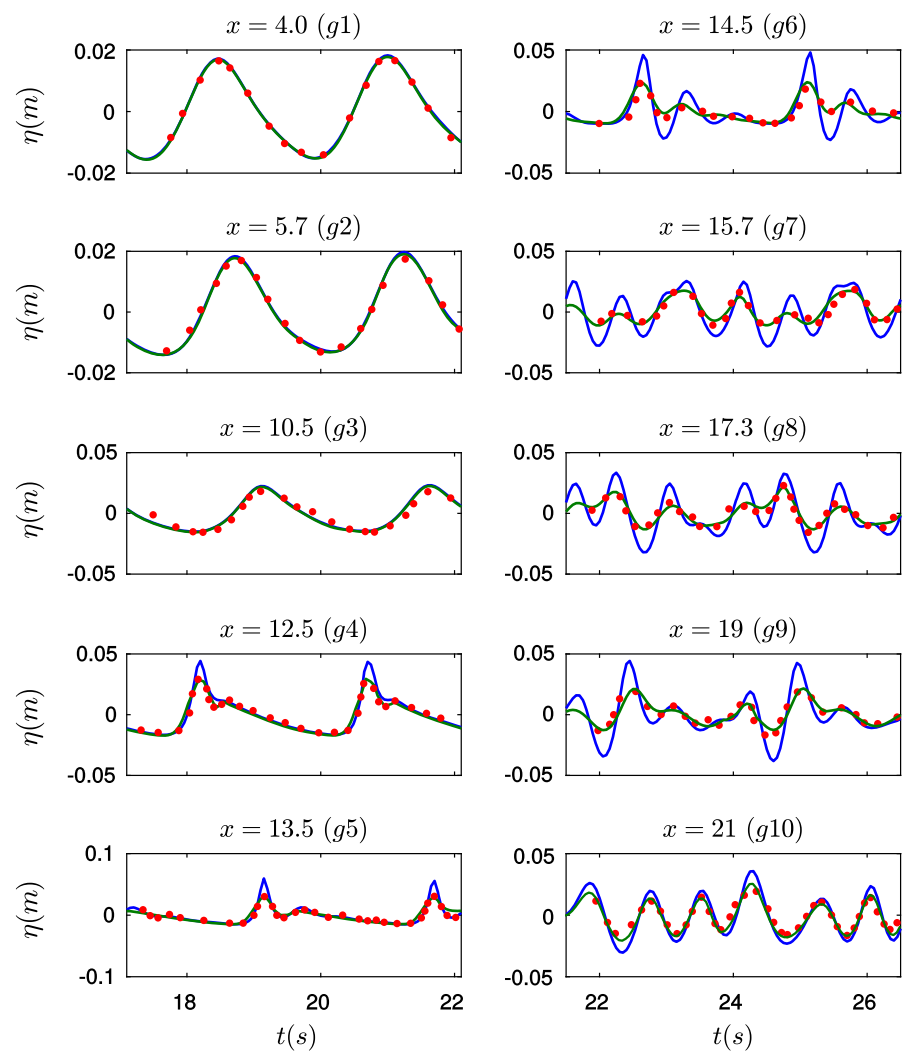

Fig. 19 Comparison of experiment data $(b)$ (red points) and simulated ones with breaking mechanism (green) and without (blue) (Color figure online)

\section{Conclusions}

In this work, a non-hydrostatic two-layer model has been proposed to incorporate dispersive effects in the propagation of waves in a homogeneous, inviscid and incompressible fluid. The presented model corresponds to the system derived by E.D.Fernández Nieto et al. in [34] for the two-layer case, with a slight difference in the definition of the non-hydrostatic pressure at the interface. This allows us to derive a two-layer non-hydrostatic model with improved dispersive properties. The advantage is that this is done while keeping the computational cost of the model low.

The numerical scheme employed combines a finite volume segment path-conservative scheme for the underlying hyperbolic system and finite differences for discretization of nonhydrostatic terms. Furthermore, it is second order accurate and it is well-balanced for the water at rest solution, positive preserving and linearly $\mathrm{L}^{\infty}$-stable under the usual CFL condition. The extension to bidimensional domains has been done. More detail about the validation of the model is carrying out.

The model is also able to simulate correctly the run-up and run-down process in wet-dry areas. Moreover, no numerical truncation for the non-hydrostatic pressure is needed at wetdry areas, where non-hydrostatic pressure vanishes. To the best of our knowledge, this is an 
improvement in non-hydrostatic numerical schemes, where usually non-hydrostatic pressure is truncated to zero up to a threshold value.

As it is well known, non-hydrostatic models need some dissipative mechanism for breaking waves to accurately model waves near the coastal areas (see [33]). We have proposed a new, efficient and straightforward breaking mechanism that performs appropriately in the tests shown.

Numerical simulations show that the approach presented here correctly solves the propagation of eulerian solitary waves of high amplitude, preserving their shape for large integration times accurately. Comparison with experimental data is also presented. Experimental data justifies the need to incorporate dispersive effects to capture faithfully waves in the vicinity of the continental shelf. Moreover, complex processes such as run-up, shoaling, the appearance of higher harmonics and wet-dry areas, that can not be well reproduced with a one-layer non-hydrostatic system, are simulated successfully for the proposed tests. We would like to stress the good performance when dealing with a wide range of non-linear dispersive waves, as it was said in Sect. 6.5, where usually a Green-Naghdi type model or multilayer nonhydrostatic model is needed to correctly simulate the tests. The system proposed in this work can deal with such dispersive waves with a two-layer system, without the need of increasing the order of the derivatives o more layers which would increase the complexity of the model and its computational cost.

Numerical tests also evince that the proposed model introduced in this work with the jump of the non-hydrostatic pressure at the interface (16), improves the results with respect to the two-layer system derived in [34]. This has been shown by comparing with analytical solutions from the Stokes first order theory. This improvement is achieved with the same computational cost than the original two-layer system derived in [34].

The two-layer model and the numerical scheme presented in this work provides thus an efficient and accurate approach to model dispersive effects in the propagation of waves near coastal areas and intermediate waters.

Acknowledgements This research has been partially supported by the Spanish Government and FEDER through Research Project MTM2015-70490-C2-1-R and MTM2015-70490-C2-2-R, and Andalusian Government Research Project P11-FQM-8179. Funding was provided by Ministerio de Economía y Competitividad and Consejería de Economía, Innovación, Ciencia y Empleo, Junta de Andalucía.

\section{A Hyperbolicity of the Underlying Hydrostatic System}

\section{A.1 Hyperbolicity}

Let us consider the underlying hydrostatic system (20) written in quasi-linear form

$$
\partial_{t} \boldsymbol{U}+\mathcal{A} \partial_{x} \boldsymbol{U}=\boldsymbol{G}(\boldsymbol{U}) \partial_{x} H,
$$

where $\mathcal{A}=\mathcal{J}_{F}+\boldsymbol{B}$, being $\mathcal{J}_{F}$ the Jacobian matrix of the flux $\boldsymbol{F}$. The characteristic polynomial of the matrix $\mathcal{A}$ is given by

$$
\mathcal{P}\left(\lambda, l_{1}\right)=\frac{1}{2}\left(u_{1}-\lambda\right)\left(u_{2}-\lambda\right) \mathcal{Q}\left(\lambda, l_{1}\right),
$$

being $\mathcal{Q}\left(\lambda, l_{1}\right)$ a third order polynomial on $\lambda$ given by

$$
\begin{aligned}
\mathcal{Q}\left(\lambda, l_{1}\right) & =f(\lambda)-R\left(l_{1}\right), \\
f(\lambda) & =\left(3 u_{1}-u_{2}-2 \lambda\right)\left(\left(u_{2}-\lambda\right)^{2}-g h\right),
\end{aligned}
$$




$$
\begin{aligned}
R\left(l_{1}\right) & =l_{1} K, \\
K & =\left(u_{1}-u_{2}\right)\left(\left(u_{1}-u_{2}\right)^{2}-4 g h\right) .
\end{aligned}
$$

For the sake of simplicity on the notation, we do not write explicitly the dependence on $\boldsymbol{U}$. Let us study the hyperbolicity of the hydrostatic system. It is easily to check that

$$
\lambda_{1}=u_{1}, \lambda_{2}=u_{2}
$$

are eigenvalues of the system for every $l_{1} \in(0,1)$. It remains to check if the cubic polynomial $\mathcal{Q}(\lambda)$ has three distinct roots.

We will prove that the cubic polynomial it has always three different roots for every $l_{1} \in[0,1]$, and in particular for every $l_{1} \in(0,1)$ as we just request. Let us give a sketch of the proof:

1. Let us remark that $\mathcal{Q}$ is a cubic polynomial on $\lambda$ satisfying

$$
\mathcal{Q}\left(-\infty, l_{1}\right)=+\infty, \mathcal{Q}\left(\infty, l_{1}\right)=-\infty .
$$

2. Note that $f(\lambda)$ is a cubic polynomial that does not depend on $l_{1}$. Moreover, it has two local extrema given by the roots of $f^{\prime}(\lambda)$ :

$$
\lambda^{ \pm}=\frac{u_{1}+u_{2}}{2} \pm \sqrt{\frac{1}{3}+\left(\frac{u_{1}-u_{2}}{2}\right)^{2}} .
$$

3. A sufficient and necessary condition for the existence of three real and distinct roots of the cubic polynomial $\mathcal{Q}\left(\lambda, l_{1}\right)$ is that:

$$
f\left(\lambda^{-}\right)<R\left(l_{1}\right) \text { and } f\left(\lambda^{+}\right)>R\left(l_{1}\right) .
$$

4. Note that when $l_{1}=0$, the polynomial $\mathcal{Q}(\lambda, 0)$ has three roots:

$$
\lambda_{3}=\frac{3 u_{1}-u_{2}}{2}, \lambda_{4,5}=u_{2} \pm \sqrt{g h},
$$

and thus $f\left(\lambda^{-}\right)<R(0)$ and $f\left(\lambda^{+}\right)>R(0)$.

Similarly, when $l_{1}=1$, the polynomial $\mathcal{Q}(\lambda, 1)$ has three roots:

$$
\lambda_{3}=\frac{-u_{1}+3 u_{2}}{2}, \lambda_{4,5}=u_{1} \pm \sqrt{g h},
$$

and therefore $f\left(\lambda^{-}\right)<R(1)$ and $f\left(\lambda^{+}\right)>R(1)$.

Thus, if we assume that $K \geq 0$, then $R(1) \geq R\left(l_{1}\right) \geq R(0)$ and therefore

$$
\begin{aligned}
& f\left(\lambda^{+}\right)>R(1) \geq R\left(l_{1}\right) \\
& f\left(\lambda^{-}\right)<R(0) \leq R\left(l_{1}\right) .
\end{aligned}
$$

If we assume that $K \leq 0$, then $R(1) \leq R\left(l_{1}\right) \leq R(0)$ and therefore

$$
\begin{aligned}
& f\left(\lambda^{+}\right)>R(0) \geq R\left(l_{1}\right) \\
& f\left(\lambda^{-}\right)<R(1) \leq R\left(l_{1}\right) .
\end{aligned}
$$

This concludes the proof. 


\section{A.2 A First Order Approximation for the Eigenvalues}

Let us denote the eigenvalues that depends on $l_{1}$ as

$$
\lambda_{1}\left(l_{1}\right)=u_{1}, \lambda_{2}\left(l_{1}\right)=u_{2},
$$

as the known eigenvalues for any $l_{1} \in(0,1)$, and

$$
\lambda_{3}\left(l_{1}\right), \lambda_{4}\left(l_{1}\right), \lambda_{5}\left(l_{1}\right)
$$

as the eigenvalues that are roots of the cubic polynomial $\mathcal{Q}\left(\lambda, l_{1}\right)$. As a particular case, we have found an explicit form of the eigenvalues for $l_{1}=1 / 2$,

$$
\lambda_{1}=u_{1}, \lambda_{2}=u_{2}, \lambda_{3}=\frac{u_{1}+u_{2}}{2}, \lambda_{4,5}=\frac{u_{1}+u_{2}}{2} \pm \sqrt{g h+\frac{3}{4}\left(u_{1}-u_{2}\right)^{2}} .
$$

Let us consider

$$
\lambda\left(l_{1}\right) \in\left\{\lambda_{3}\left(l_{1}\right), \lambda_{4}\left(l_{1}\right), \lambda_{5}\left(l_{1}\right)\right\},
$$

an eigenvalue that depends on $l_{1}$ and is a root of the cubic polynomial $\mathcal{Q}\left(\lambda, l_{1}\right)$. We propose the following approximation of the eigenvalues, that gives the exact roots of the cubic polynomial $\mathcal{Q}\left(\lambda, l_{1}\right)$ for $l_{1} \in\{0,1 / 2,1\}$.

$$
\begin{gathered}
\lambda_{3}\left(l_{1}\right) \approx \tilde{\lambda_{3}}\left(l_{1}\right)=\left(\frac{3}{2}-2 l_{1}\right) u_{1}+\left(2 l_{1}-\frac{1}{2}\right) u_{2}, \\
\lambda_{4,5}\left(l_{1}\right) \approx \widetilde{\lambda_{4,5}}\left(l_{1}\right)=l_{1} u_{1}+l_{2} u_{2} \pm \sqrt{g h+3 l_{1} l_{2}\left(u_{1}-u_{2}\right)^{2}} .
\end{gathered}
$$

Another approximation for the eigenvalues is proposed in the following. Since $\lambda\left(l_{1}\right)$ is a root of $\mathcal{Q}\left(\lambda, l_{1}\right)$, then

$$
\mathcal{Q}\left(\lambda\left(l_{1}\right), l_{1}\right)=0,
$$

and deriving with respect to $l_{1}$ it yields

$$
\lambda^{\prime}\left(l_{1}\right)=\frac{\left(\left(u_{1}-u_{2}\right)^{2}-4 g h\right)\left(u_{1}-u_{2}\right)}{2\left(g h-3 u_{1} u_{2}+3\left(u_{1}+u_{2}-\lambda\left(l_{1}\right)\right) \lambda\left(l_{1}\right)\right)} .
$$

Thus, we propose to approximate the eigenvalues that are roots of $\mathcal{Q}\left(\lambda, l_{1}\right)$ with the first order approximation

$$
\lambda_{i}\left(l_{1}\right) \approx \tilde{\lambda_{i}}=\lambda_{i}(1 / 2)+\lambda_{i}^{\prime}(1 / 2)\left(l_{1}-1 / 2\right), i \in\{3,4,5\},
$$

that can be explicitly computed, since $\lambda_{i}(1 / 2)$ are known:

$$
\begin{aligned}
\lambda_{3}\left(l_{1}\right) \approx \tilde{\lambda_{3}}= & \frac{u_{1}+u_{2}}{2}+\left(u_{1}-u_{2}\right)\left(1-2 l_{1}\right) \frac{g h-\frac{1}{4}\left(u_{1}-u_{2}\right)^{2}}{g h+\frac{3}{4}\left(u_{1}-u_{2}\right)^{2}}+\mathcal{O}\left(l_{1}^{2}\right), \\
\lambda_{4,5}\left(l_{1}\right) \approx \widetilde{\lambda_{4,5}}= & \frac{u_{1}+u_{2}}{2} \pm \sqrt{g h+\frac{3}{4}\left(u_{1}-u_{2}\right)^{2}} \\
& +\left(u_{1}-u_{2}\right)\left(l_{1}-\frac{1}{2}\right) \frac{g h-\frac{1}{4}\left(u_{1}-u_{2}\right)^{2}}{g h+\frac{3}{4}\left(u_{1}-u_{2}\right)^{2}}+\mathcal{O}\left(l_{1}^{2}\right) .
\end{aligned}
$$

This procedure is more rigorous and lead to a more sophisticated expressions of the approximated eigenvalues. 


\section{B Linear Dispersion Properties}

\section{B.1 Linear Dispersion Relation}

Substituting (23) into (24) yields the linear dispersion relation:

$$
\frac{C^{2}}{g H}=\frac{N_{0}+N_{1}(k H)^{2}}{D_{0}+D_{1}(k H)^{2}+D_{2}(k H)^{4}},
$$

where

$$
\left\{\begin{array}{l}
N_{0}=1, \quad N_{1}=\frac{l_{1} l_{2}\left(-\gamma_{1}-\gamma_{2}+2\left(\gamma_{2}-1\right) l_{1}+2\right)}{4\left(\gamma_{1}+\gamma_{2}\right)}, \\
D_{0}=1, \quad D_{1}=\frac{\gamma 1+\gamma 2+2\left(\gamma^{2}-2\right) l_{1}^{2}-2 l_{1}\left(\gamma 1+\gamma^{2}-2\right)}{4\left(\gamma 1+\gamma^{2}\right)}, \quad D_{2}=\frac{l_{1}^{2} l_{2}^{2}\left(\gamma 1-\gamma_{2}\right)}{16(\gamma 1+\gamma 2)} .
\end{array}\right.
$$

\section{Breaking Waves Parameters}

By taking into account the two vertical velocities equations in (45) and the incompressibility equations, which relates $w_{\alpha}$ with $u_{\alpha}$, lead us to write $P$ in terms of the derivatives of $U$

$A_{(x x)} \partial_{x x} \boldsymbol{U}+A_{(x t)} \partial_{x t} \boldsymbol{U}+A_{(x)} \partial_{x} \boldsymbol{U}+A_{(t)} \partial_{t} \boldsymbol{U}+A \boldsymbol{U}+B=\left(\begin{array}{c}p_{b}-p_{I} \\ \gamma_{1} p_{b}+\gamma_{2} p_{I}\end{array}\right)+I_{(\varsigma)} \partial_{x} \boldsymbol{U}$,

where

$$
I_{(\varsigma)}=\left(\begin{array}{cc}
-\varsigma_{1} l_{1} h & 0 \\
0 & -\varsigma_{2} l_{2} h
\end{array}\right), A_{(\cdot)} \in \mathcal{M}_{2}(\mathbb{R})
$$

We propose define $\varsigma_{\alpha}$ such that

$$
I_{(\varsigma)}:=\operatorname{Diag}\left(A_{(x)}\right) \text {. }
$$

We then proceed to compute $A_{(x)}$. The two continuity equations can be written as

$$
\left\{\begin{array}{l}
w_{1}=u_{1} \partial_{x} H-\frac{1}{2} l_{1} h \partial_{x} u_{1}-\partial_{t} H \\
w_{2}=-u_{1} \partial_{x} l_{1} h+u_{2} \partial_{x} z_{I}-l_{1} h \partial_{x} u_{1}-\frac{1}{2} l_{2} h \partial_{x} u_{2}-\partial_{t} H .
\end{array}\right.
$$

Neglecting mass transfer terms due to $\Gamma_{I}$, the vertical equations can be written as

$$
\left\{\begin{array}{l}
\partial_{t}\left(l_{1} h w_{1}\right)+\partial_{x}\left(l_{1} h u_{1} w_{1}\right)=p_{b}-p_{I}+\varsigma_{1} l_{1} h \partial_{x} u_{1} \\
\partial_{t}\left(l_{2} h w_{2}\right)+\partial_{x}\left(l_{2} h u_{2} w_{2}\right)=\gamma_{1} p_{b}+\gamma_{2} p_{I}+\varsigma_{2} l_{2} h \partial_{x} u_{2}
\end{array}\right.
$$


Then, retaining at the left hand side of Eq. (50) only the terms multiplied by $\partial_{x} u_{\alpha}$ at the equation concerning to the layer $\alpha$, leads to

$$
I_{(\varsigma)}=\left(\begin{array}{cc}
l_{1} h\left(-\partial_{t} l_{1} h+w_{1}-u_{1} \partial_{x}\left(l_{1} h+H\right)\right) & 0 \\
0 & l_{2} h\left(-\partial_{t} l_{2} h+w_{2}+u_{2} \partial_{x}\left(z_{I}-l_{2} h\right)\right)
\end{array}\right),
$$

Again, using that $\Gamma_{I}=0, I_{(\varsigma)}$ can be rewritten as

$$
I_{(\varsigma)}=\left(\begin{array}{cc}
l_{1} h\left(-w_{1}+3 u_{1} \partial_{x} H-2 \partial_{t} H\right) & 0 \\
0 & l_{2} h\left(-w_{2}-2 \partial_{t} H-3 u_{2} \partial_{x} z_{I}\right)
\end{array}\right),
$$

and finally we propose

$$
\left\{\begin{array}{l}
\varsigma_{1}=\left(w_{1}-3 u_{1} \partial_{x} H+2 \partial_{t} H\right) \\
\varsigma_{2}=\left(w_{2}+3 u_{2} \partial_{x} z_{I}+2 \partial_{t} H\right) .
\end{array}\right.
$$

\section{Coefficients and Matrices of the Linear System}

\section{D.1 Coefficients of the Poisson-Like Equations}

The coefficients appearing in (37) and (38) are:

$$
\begin{aligned}
& \left\{\begin{array}{l}
a_{1}=-\left(l_{1} h\right)^{2}, \\
a_{2}=-l_{1}^{2} h \partial_{x} h, \\
a_{3}=l_{1} h \partial_{x x}\left(l_{1} h+2 l_{2} h-2 \eta\right)+\partial_{x}\left(h_{1}+2 l_{2} h-2 \eta\right)^{2}+4, \\
a_{4}=-\left(l_{1} h\right)^{2}, \\
a_{5}=-l_{1} h \partial_{x}\left(3 l_{1} h+4 l_{2} h-4 \eta\right), \\
a_{6}=-l_{1} h \partial_{x x}\left(l_{1} h+2 l_{2} h-2 \eta\right)-\partial_{x}\left(l_{1} h+2 l_{2} h-2 \eta\right)^{2}-4,
\end{array}\right. \\
& \left\{\begin{array}{l}
b_{1}=-\left(l_{2} h\right)^{2}\left(\gamma_{1}+2 \frac{l_{1}}{l_{2}}\right), \\
b_{2}=l_{2} h \partial_{x}\left(\left(4-\gamma_{1}\right) l_{2} h-4 \eta\right), \\
b_{3}=l_{2} h \partial_{x x}\left(2 l_{1} h+\left(2 \gamma_{1}+3\right) l_{2} h-2\left(\gamma_{1}+2\right) \eta\right)+\gamma_{1} \partial_{x}\left(l_{2} h-2 \eta\right)+4 \gamma_{1}, \\
b_{4}=-\left(l_{2} h\right)^{2}\left(\gamma_{2}+2 \frac{l_{1}}{l_{2}}\right), \\
b_{5}=l_{2} h \partial_{x}\left(4 l_{1} h+\left(4-\gamma_{2}\right) l_{2} h-4 \eta\right), \\
b_{6}=l_{2} h \partial_{x x}\left(-2 l_{1} h+\left(2 \gamma_{2}-5\right) l_{2} h-2\left(\gamma_{2}-2\right) \eta\right)+\gamma_{2} \partial_{x}\left(l_{2} h-2 \eta\right)+4 \gamma_{2},
\end{array}\right. \\
& R H S_{1}=l_{1} h^{(\widetilde{k})} \partial_{x} q_{u, 1}^{(\widetilde{k})}-2 q_{u, 1}^{(\widetilde{k})} \partial_{x} z_{1}^{(\widetilde{k})}+2 q_{w, 1}^{(\widetilde{k})}+2 h^{(\widetilde{k})} \partial_{t} H \text {, }
\end{aligned}
$$




$$
R H S_{2}=2 l_{1} h^{(\widetilde{k})} \partial_{x} q_{u, 1}^{(\widetilde{k})}+l_{2} h^{(\widetilde{k})} \partial_{x} q_{u, 2}^{(\widetilde{k})}-2 q_{u, 2}^{(\widetilde{k})} \partial_{x} z_{2}^{(\widetilde{k})}+2 q_{w, 2}^{(\widetilde{k})}+2 h^{(\widetilde{k})} \partial_{t} H
$$

\section{D.2 Matrices of the Linear Systems}

After replace (39) and (40) in (37) and (38), one has to solve a linear system

$$
\begin{aligned}
\boldsymbol{A P} & =\mathcal{R} \mathcal{H S}, \\
\boldsymbol{A} & =\boldsymbol{D}+\boldsymbol{M}, \boldsymbol{D}=\left(\begin{array}{c|c}
T_{(1)} & \mathbf{0} \\
\hline \mathbf{0} & T_{(2)}
\end{array}\right), \quad \boldsymbol{M}=\left(\begin{array}{c|c}
\mathbf{0} & C_{(1)} \\
\hline C_{(2)} & \mathbf{0}
\end{array}\right),
\end{aligned}
$$

being $T_{(j)}, C_{(j)}$ tridiagonal matrices of dimension $N \times N$ given by:

$$
\begin{aligned}
T_{(1)} & =\frac{A_{(1)}}{\Delta x^{2}} T_{(1,-2,1)}+\frac{A_{(2)}}{2 \Delta x} T_{(-1,0,1)}+A_{(3)} I, \\
C_{(1)} & =\frac{A_{(4)}}{\Delta x^{2}} T_{(1,-2,1)}+\frac{A_{(5)}}{2 \Delta x} T_{(-1,0,1)}+A_{(6)} I, \\
T_{(2)} & =\frac{B_{(1)}}{\Delta x^{2}} T_{(1,-2,1)}+\frac{B_{(2)}}{2 \Delta x} T_{(-1,0,1)}+B_{(3)} I, \\
C_{(2)} & =\frac{B_{(4)}}{\Delta x^{2}} T_{(1,-2,1)}+\frac{B_{(5)}}{2 \Delta x} T_{(-1,0,1)}+B_{(6)} I,
\end{aligned}
$$

where

$$
T_{(a, b, c)}=\left(\begin{array}{ccccc}
b & c & & & 0 \\
a & b & c & & \\
& \ddots & \ddots & \ddots & \\
& & a & b & c \\
0 & & & a & b
\end{array}\right)
$$

gather the centred finite difference matrix of second $\left(T_{(1,-2,1)}\right)$ and first $\left(T_{(-1,0,1)}\right)$ order, and $I$ the identity matrix of dimension $2 N \times 2 N$.

The matrices $A_{(j)}$ and $B_{(j)}, j \in\{1, \ldots, 6\}$ are diagonal matrices of dimension $N \times N$

$$
A_{(j)}=\left(\begin{array}{ccccc}
a_{j, 1} & & & & 0 \\
& a_{j, 2} & & & \\
& & \ddots & & \\
0 & & & a_{j, N-1} & \\
& & & & a_{j, N}
\end{array}\right), B_{(j)}=\left(\begin{array}{ccccc}
b_{j, 1} & & & & 0 \\
& b_{j, 2} & & & \\
& & \ddots & & \\
& & & b_{j, N-1} & \\
0 & & & & b_{j, N}
\end{array}\right) \text {, }
$$

where the coefficients $a_{j, i}$ (and $b_{j, i}$ ) are the point value approximations of $a_{j}$ (and $b_{j}$ ) described in Appendix D.2. For example,

$$
\begin{aligned}
a_{3, i}= & h_{1, i} \frac{2 H_{i-1}-h_{1, i-1}-2\left(2 H_{i}-h_{1, i}\right)+2 H_{i+1}-h_{1, i+1}}{\Delta x^{2}} \\
& +\frac{2 H_{i+1}-h_{1, i+1}-\left(2 H_{i-1}-h_{1, i-1}\right)}{2 \Delta x}+4 .
\end{aligned}
$$

\section{D.3 Analysis of the Linear System for Small Water Heights}

If we assume

$$
h=\epsilon, u_{\alpha}=w_{\alpha}=0,
$$


then the coefficients (52) and (53) reduce to

$$
\left\{\begin{array} { l } 
{ a _ { 1 } = - l _ { 1 } ^ { 2 } \epsilon ^ { 2 } , } \\
{ a _ { 2 } = 0 , } \\
{ a _ { 3 } = 4 ( 1 + ( \partial _ { x } H ) ^ { 2 } ) + 2 l _ { 1 } \epsilon \partial _ { x x } H , } \\
{ a _ { 4 } = - l _ { 1 } ^ { 2 } \epsilon ^ { 2 } , } \\
{ a _ { 5 } = - 4 l _ { 1 } \epsilon \partial _ { x } H , } \\
{ a _ { 6 } = - 4 ( 1 + ( \partial _ { x } H ) ^ { 2 } ) - 2 l _ { 1 } \epsilon \partial _ { x x } H , }
\end{array} \quad \left\{\begin{array}{l}
b_{1}=-l_{2}\left(\left(\gamma_{1}-2\right) l_{2}+2\right) \epsilon^{2}, \\
b_{2}=4 l_{2} \epsilon \partial_{x} H, \\
b_{3}=4 \gamma_{1}\left(1+\left(\partial_{x} H\right)^{2}\right)+2 l_{2}\left(\gamma_{1}+2\right) \epsilon \partial_{x x} H, \\
b_{4}=-l_{2}\left(\left(\gamma_{2}-2\right) l_{2}+2\right) \epsilon^{2}, \\
b_{5}=-4 l_{2} \epsilon \partial_{x} H, \\
b_{6}=4 \gamma_{2}\left(1+\left(\partial_{x} H\right)^{2}\right)+2 l_{2}\left(\gamma_{2}-2\right) \epsilon \partial_{x x} H,
\end{array}\right.\right.
$$

and the Right Hand Side vectors reduce to

$$
\mathcal{R H S}=\left(\begin{array}{l}
\mathcal{R H S}_{1} \\
\mathcal{R H S}_{2}
\end{array}\right)=\left(\begin{array}{l}
\mathbf{0} \\
\mathbf{0}
\end{array}\right)
$$

In the following analysis we will assume:

$$
\epsilon^{2} \approx 0, \epsilon \partial_{x} H \approx 0, \epsilon \partial_{x x} H \approx 0,
$$

and for the sake of simplicity we assume that $\partial_{x} H=m$. Then the linear system becomes

$$
\boldsymbol{A}=4\left(1+m^{2}\right)\left(\begin{array}{c|c}
\boldsymbol{I} & -\boldsymbol{I} \\
\hline \gamma_{2} \boldsymbol{I} & \gamma_{1} \boldsymbol{I}
\end{array}\right) .
$$

The matrix $\boldsymbol{A}$ is invertible

$$
\boldsymbol{A}^{-1}=\frac{1}{4\left(\gamma_{1}+\gamma_{2}\right)\left(m^{2}+1\right)}\left(\begin{array}{c|c}
\gamma_{1} \boldsymbol{I} & \boldsymbol{I} \\
\hline-\gamma_{2} \boldsymbol{I} & \boldsymbol{I}
\end{array}\right),
$$

since we assume in Remark 4 that $\gamma_{1}+\gamma_{2} \neq 0$.

We note that (54) collects the particular case of a slowly varying bathymetry $\partial_{x} H \approx 0$, and in particular the case under study in this work, when $\partial_{x} H=m$ with $\epsilon \cdot m \approx 0$.

\section{References}

1. Abbott, M.B., McCowan, A.D., Warren, I.R.: Accuracy of short wave numerical models. J. Hydraul. Eng. 110(10), 1287-1301 (1984)

2. Abgrall, R., Karni, S.: A comment on the computation of non-conservative products. J. Comput. Phys. 229, 2759-2763 (2010)

3. Adsuara, J.E., Cordero-Carrión, I., Cerdá-Durán, P., Aloy, M.A.: Scheduled relaxation jacobi method: Improvements and applications. J. Comput. Phys. 321, 369-413 (2016)

4. Ai, C., Jin, S.: A multi-layer non-hydrostatic model for wave breaking and run-up. Coast. Eng. 62, 1-8 (2012)

5. Ai, C., Jin, S., Lv, B.: A new fully non-hydrostatic $3 \mathrm{~d}$ free surface flow model for water wave motions. Int. J. Numer. Methods Fluids 66(11), 1354-1370 (2010)

6. Aïssiouene, N., Bristeau, M.-O., Godlewski, E., Mangeney, A., Parés, C., Sainte-Marie, J.: Application of a combined finite element-finite volume method to a 2D non-hydrostatic shallow water problem. In: Cancés, C., Omnes, P. (eds.) Finite Volumes for Complex Applications VIII-Hyperbolic, Elliptic and Parabolic Problems, pp. 219-226. Springer, Cham (2017) 
7. Audusse, E., Bristeau, M.O., Perthame, B., Sainte-Marie, J.: A multilayer Saint-Venant system with mass exchanges for shallow water flows. Derivation and numerical validation. ESAIM Math. Model. Numer. Anal. 45, 169-200 (2011). 01

8. Bai, Y., Cheung, K.F.: Depth-integrated free-surface flow with a two-layer non-hydrostatic formulation. Int. J. Numer. Methods Fluids 69(2), 411-429 (2011)

9. Bai, Y., Cheung, K.F.: Depth-integrated free-surface flow with parameterized non-hydrostatic pressure. Int. J. Numer. Methods Fluids 71(4), 403-421 (2012)

10. Bai, Y., Cheung, K.F.: Dispersion and kinematics of multi-layer non-hydrostatic models. Ocean Model. 92, 11-27 (2015)

11. Bai, Y., Cheung, K.F.: Linear shoaling of free-surface waves in multi-layer non-hydrostatic models. Ocean Model. 121, 90-104 (2018)

12. Beji, S., Battjes, J.A.: Experimental investigation of wave propagation over a bar. Coast. Eng. 19, 151-162 (1993)

13. Berthon, C., Coquel, F., LeFloch, P.G.: Why many theories of shock waves are necessary: kinetic functions, equivalent equations, and fourth-order models. J. Comput. Phys. 227, 4162-4189 (2008)

14. Bonneton, P., Chazel, F., Lannes, D., Marche, F., Tissier, M.: A splitting approach for the fully nonlinear and weakly dispersive Green-Naghdi model. J. Comput. Phys. 230(4), 1479-1498 (2011)

15. Boussinesq, J.: Théorie des ondes et des remous qui se propagent le long dun canal rectangulaire horizontal, en communiquant au liquide contenu dans ce canal des vitesses sensiblement pareilles de la surface au fond. J. Math. Pures Appl. 17, 55-108 (1872)

16. Bristeau, M.-O., Mangeney, A., Sainte-Marie, J., Seguin, N.: An energy-consistent depth-averaged euler system: derivation and properties. Discrete Continuous Dyn. Syst. Ser. B 20(4), 961-988 (2015)

17. Castro, M.J., Fernández-Nieto, E.D.: A class of computationally fast first order finite volume solvers: PVM methods. SIAM J. Sci. Comput. 34(4), 173-196 (2012)

18. Castro, M.J., Ferreiro, A.M.F., García-Rodríguez, J.A., González-Vida, J.M., Macías, J., Parés, C., Vázquez-Cendón, M.E.: The numerical treatment of wet/dry fronts in shallow flows: application to onelayer and two-layer systems. Math. Comput. Model. 42(3), 419-439 (2005)

19. Castro, M.J., Gallardo, J.M., Parés, C.: High order finite volume schemes based on reconstruction of states for solving hyperbolic systems with nonconservative products. Applications to shallow water systems. Math. Comput. 75, 1103-1134 (2006)

20. Castro, M.J., Pardo, A., Parés, C., Toro, E.F.: On some fast well-balanced first order solvers for nonconservative systems. Math. Comput. 79(271), 1427-1472 (2010)

21. Castro, M.J., Morales de Luna, T., Parés, C.: Chapter 6-well-balanced schemes and path-conservative numerical methods. In: Abgrall, Rémi, Shu, C.-W. (eds.) Handbook of Numerical Methods for Hyperbolic Problems, volume 18 of Handbook of Numerical Analysis, pp 131-175. Elsevier, Amsterdam (2017)

22. Casulli, V.: A semi-implicit finite difference method for non-hydrostatic free surface flows. Numer. Methods Fluids 30(4), 425-440 (1999)

23. Casulli, V., Zanolli, P.: Semi-implicit numerical modeling of nonhydrostatic free-surface flows for environmental problems. Math. Comput. Model. 36(9), 1131-1149 (2002)

24. Cea, L., Stelling, G., Zijlema, M.: Non-hydrostatic 3D free surface layer-structured finite volume model for short wave propagation. Int. J. Numer. Methods Fluids 61(4), 382-410 (2008)

25. Chazel, F., Lannes, D., Marche, F.: Numerical simulation of strongly nonlinear and dispersive waves using a Green-Naghdi model. J. Sci. Comput. 48(1), 105-116 (2011)

26. Clamond, D., Dutykh, D. https://es.mathworks.com/matlabcentral/fileexchange/39189-solitary-waterwave (2012)

27. Cui, H., Pietrzak, J.D., Stelling, G.S.: Optimal dispersion with minimized poisson equations for nonhydrostatic free surface flows. Ocean Model. 81, 1-12 (2014)

28. Dingemans, M.W.: Comparison of computations with Boussinesq-like models and laboratory measurements. Report H-1684.12, 32, Delft Hydraulics (1994)

29. Duran, A., Marche, F.: A discontinuous galerkin method for a new class of Green-Naghdi equations on simplicial unstructured meshes. Appl. Math. Model. 45, 840-864 (2017)

30. Dutykh, D., Clamond, D.: Efficient computation of steady solitary gravity waves. Wave Motion 51(1), 86-99 (2014)

31. Enet, F., Grilli, S.T.: Experimental study of tsunami generation by three-dimensional rigid underwater landslides. J. Waterw. Port Coast. Ocean Eng. 133(6), 442-454 (2007)

32. Escalante, C., Macías, J., Castro, M. J.: Performance assessment of tsunami-HySEA model for NTHMP tsunami currents benchmarking. Part I lab data. Coast. Eng. (2017)

33. Escalante, C., Morales, T., Castro, M.J.: Non-hydrostatic pressure shallow flows: GPU implementation using finite volume and finite difference scheme. Appl. Math. Comput. 338, 631-659 (2018) 
34. Fernández-Nieto, E.D., Parisot, M., Penel, Y., Sainte-Marie, J.: Layer-averaged approximation of Euler equations for free surface flows with a non-hydrostatic pressure. Commun. Math. Sci. (hal-01324012v3) (2018)

35. Fernández-Nieto, E.D., Koné, E.H., Chacón Rebollo, T.: A multilayer method for the hydrostatic NavierStokes equations: a particular weak solution. J. Sci. Comput. 60(2), 408-437 (2014)

36. Fernández-Nieto, E.D., Koné, E.H., Rebollo, T.: Chacón: A multilayer method for the hydrostatic NavierStokes equations: a particular weak solution. J. Sci. Comput. 60, 408-437, 08 (2014)

37. Gallerano, F., Cannata, G., Lasaponara, F., Petrelli, C.: A new three-dimensional finite-volume nonhydrostatic shock-capturing model for free surface flow. J. Hydrodyn. Ser. B 29(4), 552-566 (2017)

38. Gobbi, M.F., Kirby, J.T., W, G.: A fully nonlinear boussinesq model for surface waves. Part 2. Extension to o(kh)4. J. Fluid Mech. 405, 181-210 (2000)

39. Gottlieb, S., Shu, C.-W.: Total variation diminishing Runge-Kutta schemes. Math. Comput. 67(221), 73-85 (1998)

40. Green, A., Naghdi, P.: A derivation of equations for wave propagation in water of variable depth. Fluid Mech. 78, 237-246 (1976)

41. Harten, A., Lax, P.D., van Leer, B.: On upstream differencing and Godunov-type schemes for hyperbolic conservation laws. SIAM Rev. 25(1), 35-61 (1983)

42. Jeschke, A., Pedersen, G.K., Vater, S., Behrens, J.: Depth-averaged non-hydrostatic extension for shallow water equations with quadratic vertical pressure profile: equivalence to Boussinesq-type equations. Int. J. Numer. Methods Fluids 84(10), 569-583 (2017)

43. Kazolea, M., Delis, A.I., Synolakis, C.E.: Numerical treatment of wave breaking on unstructured finite volume approximations for extended Boussinesq-type equations. J. Comput. Phys. 271, 281-305 (2014)

44. Kurganov, A., Petrova, G.: A second-order well-balanced positivity preserving central-upwind scheme for the Saint-Venant system. Commun. Math. Sci. 5(1), 133-160, 03 (2007)

45. Lannes, D., Marche, F.: A new class of fully nonlinear and weakly dispersive Green-Naghdi models for efficient 2D simulations. J. Comput. Phys. 282, 238-268 (2015)

46. Lynett, P., Liu, P.L.F.: A two-layer approach to wave modelling. Proc. R. Soc. Lond. A Math. Phys. Eng. Sci. 460(2049), 2637-2669 (2004)

47. Lynett, P.J., Liu, P.L.-F.: Linear analysis of the multi-layer model. Coast. Eng. 51, 439-454 (2004)

48. Lynett, P.J., Wu, T.R., Liu, P.L.-F.: Modeling wave runup with depth-integrated equations. Coast. Eng. 46(2), 89-107 (2002)

49. Lynett, P.J., Gately, K., Wilson, R., Montoya, L., Arcas, D., Aytore, B., Bai, Y., Bricker, J.D., Castro, M.J., Cheung, K.F., David, C.G., Dogan, G.G., Escalante, C., González-Vida, J.M., Grilli, S.T., Heitmann, T.W., Horrillo, J., Knolu, U., Kian, R., Kirby, J.T., Li, W., Macías, J., Nicolsky, D.J., Ortega, S., Pampell-Manis, A., Park, Y.S., Roeber, V., Sharghivand, N., Shelby, M., Shi, F., Tehranirad, B., Tolkova, E., Thio, H.K., Veliolu, D., Yalner, A.C., Yamazaki, Y., Zaytsev, A., Zhang, Y.J.: Inter-model analysis of tsunami-induced coastal currents. Ocean Model. 114, 14-32 (2017)

50. Ma, G., Shi, F., Kirby, J.T.: Shock-capturing non-hydrostatic model for fully dispersive surface wave processes. Ocean Model. 43, 22-35 (2012)

51. Ma, G., Shi, F., Kirby, J.T.: Shock-capturing non-hydrostatic model for fully dispersive surface wave processes. Ocean Model. 43-44, 22-35 (2012)

52. Madsen, P.A., Sorensen, O.R.: A new form of the boussinesq equations with improved linear dispersion characteristics. Part 2: A slowing varying bathymetry. Coast. Eng. 18, 183-204, (1992)

53. Madsen, P.A., Bingham, H.B., Schffer, H.A.: Boussinesq-type formulations for fully nonlinear and extremely dispersive water waves: Derivation and analysis. Proc. Math. Phys. Eng. Sci. 459(2033), 10751104 (2003)

54. Muoz-Ruiz, M.: On a non-homogeneous bi-layer shallow-water problem: smoothness and uniqueness results. Nonlinear Anal. Theory Methods Appl. 59, 11 (2004)

55. Muoz-Ruiz, M.: On a nonhomogeneous bi-layer shallow-water problem: an existence theorem. Differ. Integral Equ. 17(9-10), 1175-1200 (2004)

56. Muoz-Ruiz, M.L., Chatelon, F.J., Orenga, P.: On a bi-layer shallow-water problem. Nonlinear Anal. Real World Appl. 4, 139-171 (2003)

57. Narbona-Reina, G., Zabsonré, J.D.D., Fernández-Nieto, E.D., Bresch, D.: Derivation of a bilayer model for shallow water equations with viscosity. Numerical validation. Comput. Model. Eng. Sci. 43(1), 27-71 (2009)

58. Nwogu, O.: An alternative form of the boussinesq equations for nearshore wave propagation. Waterw. Port Coast. Ocean Eng. 119, 618-638 (1994)

59. Peregrine, D.H.: Long waves on a beach. Fluid Mech. 27(4), 815-827 (1967)

60. Ricchiuto, M., Filippini, A.G.: Upwind residual discretization of enhanced Boussinesq equations for wave propagation over complex bathymetries. J. Comput. Phys. 271, 306-341 (2014) 
61. Roeber, V., Cheung, K.F., Kobayashi, M.H.: Shock-capturing Boussinesq-type model for nearshore wave processes. Coast. Eng. 57, 407-423 (2010)

62. Schffer, H.A., Madsen, P.A.: Further enhancements of Boussinesq-type equations. Coast. Eng. 26(1), 1-14 (1995)

63. Stansby, P.K., Zhou, J.G.: Shallow-water flow solver with non-hydrostatic pressure: 2 D vertical plane problems. Int. J. Numer. Methods Fluids 28(3), 541-563 (1998)

64. Stelling, G., Zijlema, M.: An accurate and efficient finite-difference algorithm for non-hydrostatic freesurface flow with application to wave propagation. Int. J. Numer. Methods Fluids 43(1), 1-23 (2003)

65. Synolakis, C.E.: The runup of solitary waves. Fluid Mech. 185, 523-545 (1987)

66. Van Leer, B.: Towards the ultimate conservative difference scheme. V. A second order sequel to Godunov's method. Comput. Phys. 32, 101-136 (1979)

67. Wei, G., Kirby, J.T., Grilli, S.T., Subramanya, R.: A fully nonlinear Boussinesq model for surface waves. Part 1. Highly nonlinear unsteady waves. J. Fluid Mech. 294(-1), 71 (1995)

68. Whitham, G.B., Wiley, : Linear and nonlinear waves. Earthq. Eng. Struct. Dyn. 4(5), 518-518 (1976)

69. Witting, J.M.: A unified model for the evolution nonlinear water waves. J. Comput. Phys. 56(2), 203-236 (1984)

70. Wu, C.H., Young, C.-C., Chen, Q., Lynett, P.J.: Efficient nonhydrostatic modeling of surface waves from deep to shallow water. J. Waterw. Port Coast. Ocean Eng. 136(2), 104-118 (2010)

71. Yamazaki, Y., Kowalik, Z., Cheung, K.F.: Depth-integrated, non-hydrostatic model for wave breaking and run-up. Numer. Methods Fluids 61, 473-497 (2008)

72. Zijlema, M., Stelling, G.S.: Further experiences with computing non-hydrostatic free-surface flows involving water waves. Int. J. Numer. Methods Fluids 48(2), 169-197 (2005) 